\title{
Proceedings of the Association of British Neurologists, St. Mary's Hospital (Imperial College), London, 5-6 September 1991
}

\section{Platform presentations}

THE RISK OF MULTIPLE SCLEROSIS AFTER A SINGLE CLINICAL EPISODE OF SUSPECTED DEMYELINATION: A FTVE YEAR FOLLOW-UP STUDY

SP Morrissey, DH Miller, WI McDonald. Institute of Neurology, London, UK

To ascertain the prognostic significance of cerebral MRI abnormalities at the presentation of a clinically isolated episode (optic neuritis, brainstem syndrome or an isolated spinal cord) we performed a follow-up study on 74 patients, after a mean of five years.

There were 44 females, 30 males, aged 13-50 years (mean age 31 years) with optic neuritis (37), brainstem (13) or spinal cord syndromes (24). All MRI scans were performed on a Picker 0.5T ( $\mathrm{SE}_{2000}$ sequence, axial contiguous slices).

At presentation, 50 patients $(68 \%)$ had an abnormal MRI with one or more cerebral white matter lesions. At follow-up: (1) normal MRI at presentation: two of $\mathbf{2 4}$ (8\%) developed multiple sclerosis on clinical grounds and seven developed MRI abnormalities. (2) Abnormal MRI at presentation: 38 of $50(76 \%)$ developed multiple sclerosis on clinical grounds, 43 developed new MRI lesions.

MRI findings at presentation are significantly predictive of the risk of subsequent progression to multiple sclerosis within five years. (Relative risk of abnormal MRI $=22$, $\chi^{2}=29, \mathrm{p}<0.001$.)

DNA SEQUENCE ANALYSIS IDENTIFIES REGIONS LIKELY TO BE RESPONSIBLE FOR NEUROTROPISM OF HIV-1

I Weber, $M$ Vallée, I Schuller, A Rolfs. Free University of Berlin, Berlin, Germany

Neurological syndromes have frequently been described in HIV-1 infected patients. Interestingly, neurological manifestations have been reported independently from the clinical stage of infected patients. Concomitantly, biological behaviour of HIV-1 isolates from the same patient differs depending on whether the virus was isolated from the cerebrospinal fluid (CSF) or the peripheral blood (PS). Mutations in the DNA of the virus might be responsible for such differing biological behaviour and might explain neurotropism of a selected set of HIV-1 isolates.

Using the polymerase chain reaction (PCR), the complete HIV-1 genome (9800 bp) can be amplified in about 25 reactions and analysed by direct sequencing of the amplification products. This approach requires about four days for the analysis of both CSF and PB isolates from one patient.

Our first sequence results of CSF and blood HIV-1 isolates from patients with severe neurological disturbances indicate that there are nucleotide changes in the envelope (env) gene more frequently from CSF isolates than from blood isolates. In these patients, most mutations were found in the 3'region of the env gene. Mutations in this region are very likely associated with the development of HIV-1 neurotropism.

EFFECT OF ABSENCE SEIZURES ON CEREBRAL OPIOID BINDING, MEASURED WITH POSITRON EMISSION TOMOGRAPHY

JS Duncan, PA Bartenstein, VJ Cunningham, DR Fish, SK Luthra, GV Sawle, RSJ Frackowiak, DJ Brooks. Institute of Neurology and MRC Cyclotron Unit, London, UK

The pathophysiological basis of generalised epilepsy is uncertain. There is evidence from experimental models that endogenous opioids may be released at the time of seizures and may have an antiepileptic effect.

We performed PET scans, using the opioid ligand ${ }^{11} \mathrm{C}$-Diprenorphine, in eight patients with primary generalised epilepsy and in eight normal subjects. Regional cerebral blood flow (rCBF) was measured interictally with $\mathrm{C}^{15} \mathrm{O}_{2}$, after which $300-500$ $\mathrm{MBq}$ of ${ }^{11} \mathrm{C}$-Diprenorphine was injected and a 90 minute dynamic study carried out. Serial absences were precipitated by hyperventilation for 10 minutes, 30-40 minutes after injection of diprenorphine. All subjects had continuous EEG monitoring.

No subject had any focal abnormalities of rCBF. In the patients, EEG showed generalised spike-wave discharges for $10-51 \%$ of the activation period. Analysis of time-activity curves and fitting of the data to a three-compartment kinetic model indicated decreases of available opioid receptors of $15-40 \%$ in brainstem, posterior cingulate, lateral parietal, lateral temporal, prefrontal and premotor cortex, but not in other sites, following generalised absences.

These results suggest that endogenous opioids are released in the brainstem and neocortex during serial absences. This may indicate the activation of an endogenous antiepileptic mechanism.

PROGRESSIVE MULTIFOCAL

LEUKOENCEPHALOPATHY IN AIDS: A

CLINICOPATHOLOGIC STUDY USING IN-SITU HYBRIDISATION AND IMMUNOHISTOCHEMISTRY

RW Gräfin von Einsiedel, TD Fife, AJ Aksamit, ME Cornford, U Tomiyasu, HH Itabashi, HV Vinters. University Hospital, Heidelberg, Germany; UCLA, Los Angeles, and Mayo Clinic, Minnesota, USA
The clinical, radiographic, and pathologic features of 15 patients with AIDS and progressive multifocal leukoencephalopathy (PML) were reviewed. Brain tissue from 10 autopsy and six biopsy specimens was studied using: in situ hybridisation for JC virus (JCV), immunohistochemistry for human immunodeficiency virus (HIV) p24 antigen, and electron microscopy. Thirteen patients presented with focal neurologic deficits, two presented with only a rapid decline in mental status. PML was commonly the initial opportunistic infection of AIDS and produced hemiparesis, dementia, dysarthria, cerebellar abnormalities, and seizures. MRI was more sensitive than CT and often showed multifocal areas of PML. CD4+ $T$ cell counts were uniformly low (mean $84 / \mathrm{mm}^{3}$ ), except in one patient who improved on AZT (3'-azido-3'-deoxythymidine; zidovudine). PML involved the cerebral hemispheres, brainstem, cerebellum, and cervical spinal cord. The distribution of brain involvement was consistent with haematogenous dissemination of the virus. In two brain specimens, multiple HIV type giant cells (HIV-GCs) were present within the regions involved by PML. When coinfection by HIV and papovavirus was present, PML dominated the pathological picture. In situ hybridisation for JCV showed virus in the nuclei of oligodendrocytes and astrocytes. Occasionally there was staining for JCV DNA in the cytoplasm of glial cells and in the neuropil, the latter possibly a correlate of papovavirus spread between myelin sheaths, as identified by electron microscopy. In situ hybridisation demonstrated more extensive foci of PML than did routine light microscopy.

THE ROLE OF OPEN BRAIN BIOPSY IN ENCEPHALITIS OF UNKNOWN ORIGIN W Reith, E Hund, D Krieger, RW Gräfin von Einsiedel, FK Albert, R v Kummer. University Hospital, Heidelberg, Germany

Chronic encephalitis often constitutes a major diagnostic problem due to the wide spectrum of possible etiologies. To determine the adequate treatment as early as possible, we performed an open brain biopsy in five unclarified cases. In three cases histological examination led to definite diagnoses. One AIDS case with two different cerebral lesions at the same time was. clinically diagnosed as Toxoplasma gondii but one lesion decreased in size after treatment. The other lesion was then biopsied and turned out to be a lymphoma. In a second case, clinically suspicious of neoplasm, biopsy material revealed cerebral cysticercosis. In a third case, the biopsy confirmed the clinical diagnosis of cerebral tuberculosis. In two of the five cases, a specific diagnosis could not be established. 
No complications were observed after the operation.

Open brain biopsy is considered as a safe method to clarify encephalitis of unknown origin. Reported complication rates are generally less than $1 \%$ if the procedure is performed by an experienced neurosurgeon. The diagnostic yield depends on the exten of the biopsy, size of the pathological process, and sophisticated planning of the biopsy site. Recent series confirmed that a definite diagnosis can be made in $50-60 \%$ of patients. Therefore, we consider brain biopsy as a useful method in encephalitis of unknown origin.

OUTCOME FOLLOWING RESECTIVE SURGERY FOR TEMPORAL LOBE EPILEPSY

RDC Elwes, G Dunn, CD Binnie, CE Polkey. The Maudsley Hospital, London, UK

The long-term outcome has been assessed in a consecutive series of 102 cases undergoing resective temporal lobe surgery because of medically intractable epilepsy. Patients were followed prospectively for a median of 61 months. Actuarial statistics were used to measure the temporal patterns of remission and stability of outcome over prolonged periods of observation. The probability of achieving one-year remission was $57 \%$ by one year, $70 \%$ by two years, and $77 \%$ by seven years. Once a patient was in one year remission the probability of remaining seizure free was $90 \%$. This rose to $94 \%$ after two consecutive years seizure free. Most patients who are going to remit following surgery have done so by two years of follow-up. Outcome at the end of the second post-operative year is a good predictor of long-term prognosis.

FOCAL LESIONS IN CREUTZFELDT-JAKOB DISEASE

N Heye, J Cervós-Navarro, H Przuntek. Free University, Berlin and Ruhr University, Bochum, Germany

There is evidence that the infectious agent of Creutzfeldt-Jakob disease (CJD) spreads via an axonal-transsynaptic pathway. Accordingly focal deficits and right/left asymmetry are common in clinical and electrophysiological findings. This has escaped attention in neuropathological studies.

To identify early, focal, or lateralised changes in CJD, we correlated the clinical and electrophysiological findings with the autopsy material in 13 cases with CJD.

In the prodromal stage signs of focal involvement were observed clinically in five cases and in eight cases in the fully developed stage. Clinical signs were generalised seven months after the beginning. In prodromal and fully developed stage nine cases showed focal abnormality in EEG records. In the terminal stage six cases had lateralised EEG findings. Beyond 16 months no focal EEG alterations were found. Four cases with a disease duration shorter than 12 months had asymmetric pathological alterations. Cases who were symptomatic longer than 12 months had generalised, uniform tissue alterations.

It can be claimed that the infectious agent spreads through the entire brain within 12 months after the initial symptoms.
FACTORS PREDICTIVE OF A GOOD RESPONSE TO TEMPORAL NEOCORTICECTOMY IN TEMPORAL LOBE EPILEPSY

D McMackin, Peng Shu Ming, J Phillips, $H$ Staunton. Richmond Institute for Neurology and Neurosurgery, Dublin, Eire

An attempt was made to define the factors predictive of a good result to temporal neocorticectomy, with preservation of amygdala and hippocampus, in the management of intractable temporal lobe epilepsy. The long term results, over a period between three and 15 years, were studied in 50 patients. Preoperative EEG assessments were based solely on interictal spikes. Three factors emerged as predictive of a good outcome: (1) a clear, repeatable anterior-mid temporal focus $(p<0.01)$, (2) stereotyped onset of the temporal lobe seizure $(p<0.05)$ and (3) greater volume of tissue removed at surgery $(p<0.05)$. On the basis of factors 1 and 2 above, namely, interictal activity and experiential phenomena at onset of seizure, the patients were divided into two groups. Group A $(n=22)$ had a stereotyped aura at seizure onset and unilateral anterior to midtemporal spike. Group B ( $n=27)$ had bilateral or extratemporal/inconsistent spike morphology and/or paraethesiae, an immediate arrest reaction or no warning at seizure onset. Those in group $A$ had a $91 \%$ chance of falling into Crandall's class 1 or 2 (cure or near cure). In those patients comprising group B only $26 \%$ achieved Crandall grouping 1 or 2, while the remaining $74 \%$ experienced an unfavourable outcome (Crandall III, IV and V). Neocorticectomy, with preservation of deeper structures and consequent implications for memory, is an effective treatment for carefully selected subjects with intractable temporal lobe epilepsy. Such selection can be performed with relatively simple technology.

NEUROLOGICAL COMPLICATIONS FOLLOWING HEART TRANSPLANTATION

WJK Cumming, D Thompson. Withington and Wythenshawe Hospitals, Manchester, UK

Between April 1987 and January 1990, 68 heart transplants were performed at Wythenshawe Hospital; 20 patients had significant neurological complications after transplantation.

Three patients developed epilepsy in the early postoperative period-one a previously undiagnosed epileptic, one in a patient with MELAS syndrome and, in one, secondary to a postoperative cerebral embolus. One patient had a period of unexplained unconsciousness, requiring intubation and ventilation and has since made a complete recovery. Three patients had a lateral popliteal nerve palsy due to trauma around the time of operation: all are recovering although one patient needed to wear a splint for 12 months before complete recovery.

Cyclosporin therapy accounted for a range of neurological complications in a high proportion of our patients. Some amounted to exacerbation of pre-existing disease (epilepsy, cerebrovascular disease, visual disturbance, migraine) and others developed de novo after initiation of cyclosporin therapy. These included muscle cramps, peripheral paraethesiae and dysaesthesiae, facial hyperaesthesia, hirsutism, gingival hyperplasia, tremor, dizzy spells, and headaches.

Neurological sequelae were common in this series of transplant patients and many of these were related, directly or indirectly, to cyclosporin therapy.

HEMISPHERIC BRAIN INFARCTIONS AND CLINICAL OUTCOME IN EARIY AND DELAYED MIDDLE CEREBRAL ARTERY RECANALISATION EB Ringelstein, R Biniek, B Ammeling, P Nolte. University Hospital, Aachen, Germany

To evaluate the influence of time of recanalisation and/or degree of initial leptomeningeal collateral blood flow in middle cerebral artery (MCA) occlusion on infarct size and clinical outcome, a series of 34 consecutive patients with acute stroke with MCA occlusions were studied by CT, initial arteriography $(n=21)$, repetitive close-meshed transcranial Doppler (TCD) ultrasonography, and a neurological stroke scale.

Type and size of infarct dependent on the location of the occluding lesions within the MCA trunk. A total of $65 \%$ of patients showed recanalisation of the occluded MCA within one week. Subsequent hyperperfusion was seen in 38 to $44 \%$ of cases. There was a weakly significant relation between size of infarction and recanalisation time within the first 20 hours. As soon as recanalisation time was greater than eight hours, lesions always extended to the cortex. Good leptomeningeal collateral blood flow significantly reduced the size of the infarct $(p<0.05)$ and significantly improved clinical outcome after 17 days and 10 months $(p<0.01)$.

Early recanalisation of embolic MCA occlusions within up to eight hours in conjunction with good transcortical collateralisation seems to have a favourable impact on infarct size and outcome, and, presumably constitutes the therapeutic time window.

TIAS: WHICH PATIENTS ARE AT HIGH (AND LOW) RISK OF FURTHER VASCULAR EVENTS? GJ Hankey, JM Slattery, CP Warlow. University of Edinburgh, Edinburgh, UK

Although, on average, patients with transient ischaemic attacks (TIA) have an increased risk of stroke and other serious vascular events, the prognosis is variable; some patients have a benign prognosis and others a poor prognosis. The aim of this study was to determine which clinical features identify patients with TIA who are at high risk (and low risk) so that effective higher risk and more costly treatments (such as carotid endarterectomy) can be targeted to the higher risk patients. In addition, lower risk and less expensive treatments may be directed to a much wider range of patients. A cohort of 469 patients with TIA, without prior stroke, were evaluated prospectively and followed up over a mean period of 4.1 years. The major outcome events of interest were (a) stroke, (b) coronary event and (c) stroke, myocardial infarction, and vascular death. The survival data were analysed using the actuarial Kaplan-Meier technique and prognostic factors were identified using the Cox proportional hazards analysis. A hazard ratio (HR) for each outcome event was 
produced for each potential prognostic factor. The adverse prognostic factors for stroke were an increasing number of TIAs in the three months before presentation (HR 1.02 , for each TIA), increasing age (HR $1 \cdot 5$, per decade), peripheral vascular disease (HR 2.3), TIAs of the brain (compared with the eye) (HR 2.3), and left ventricular hypertrophy (HR 2.2); the prognostic factors for coronary event were increasing age (HR 2.1, per decade), a combination of carotid and vertebrobasilar TIAs (HR 3.9), ischaemic heart disease (HR 2.8) and male sex (HR 2.8); and for stroke, myocardial infarction, or vascular death they were increasing age (HR 1.8, per decade), peripheral vascular disease (HR 2.4), increasing number of TIAs in the three months before presentation (HR 1.01, for each TIA), male sex (HR 2.0), a combination of carotid and vertebrobasilar TIAs (HR 2.1), TIAs of the brain (compared with the eye) (HR 1.8), and the presence of residual signs after the TIA (2.0). Before it can be concluded that these features accurately predict prognosis and can be generalised to other populations, their predictive power needs to be validated in other, independent samples of patients with TIA (which we are currently doing).

\section{RECOVERY FROM STRIATOCAPSULAR MOTOR} STROKE

C Weiller, F Chollet, RSJ Frackowiak. University of Essen, Essen, Germany and MRC Cyclotron Unit, Hammersmith Hospital, London, UK

We used PET to study changes in the functional reorganisation of the brain in 10 patients recovering from striatocapsular motor strokes compared with 10 normal subjects. Comparisons of cerebral blood flow (CBF) maps at rest showed a significantly decreased $\mathrm{CBF}$ in the patients in the basal ganglia, thalamus, sensorimotor, insular, anterior cingulate and dorsolateral prefrontal cortices, and brainstem contralateral to the recovered hand and in ipsilateral cerebellum, reflecting the distribution of dysfunction caused by the ischemic lesions. CBF was significantly increased in contralateral posterior cingulate cortex and ipsilateral premotor cortex and caudate nucleus. During finger opposition of the recovered hand the patients activated the contralateral motor areas and ipsilateral cerebellum to the same extent as normals. They activated more than normals bilateral insula, area 40, prefrontal and anterior cingulate cortices and ipsilateral premotor cortex, and basal ganglia and contralateral cerebellum. The activation pattern was also not normal when the hand contralateral to the hemiplegia was moved. Bilateral activation of motor pathways and recruitment of additional motor areas are involved in the recovery process. Activation of anterior and posterior cingulate and prefrontal cortices indicates the need for selective attention and internal cues for this otherwise simple motor task.

\section{DETECTION OF EBNA2-MRNA IN PRIMARY} CENTRAL NERVOUS SYSTEM LYMPHOMAS IN PATIENTS WITH AIDS

A Rolfs, I Weber, I Schuller, Th Dissmann, $\mathrm{K}$ Weigel. Free University of Berlin, Berlin, Germany

The high correlation of primary intracerebral non-Hodgkin lymphomas (PINHL) in patients with AIDS with the presence of Epstein-Barr virus (EBV) led us to examine the question whether DNA and RNA EBV-genomes could be detected in stereotactic brain biopsy specimens from HIV-1 infected subjects with PINHL.

Three HIV-1 positive patients with cerebral mass lesions of unknown etiology were subjected to a CT-guided stereotactic brain biopsy. Brain biopsy specimens, CSF obtained by ventricular puncture and blood were collected. The material was used to isolate DNA and RNA. The samples were analysed by polymerase chain reaction (PCR) for the presence of EBV-, CMV-, HIV-1-DNA/RNA and herpes simplex virus type I DNA (HSV). All PCR assays were performed with identical amounts of DNA or cDNA aliquots.

The histological and immunohistological findings consistently verified the diagnosis of a primary intracerebral B-cell lymphoma. Both EBNA1 and EBNA2 of EBV are detectable in the biopsy. In contrast to EBNA1, EBNA2 is overexpressed in the biopsy and the CSF cells. A semiquantitative analysis of the amplification products revealed a 15-20-fold greater amount of EBNA1-and EBNA2-specific DNA than $\beta$ actin DNA in cells of the biopsy, blood and CSF.

The results are indicative of active EBV transcription in intracerebral B-cell lymphomas. The markedly higher amounts of EBV-specific RNA found in the biopsy and CSF suggest an active transcription in the mass lesions rather than a latent inactive EBV carrier status. Detection of EBV-DNA and RNA in PINHL suggests that EBV is associated with B-cell lymphomas.

BONE MARROW TRANSPLANTATION IN JUVENILE METACHROMATIC LEUCODYSTROPHY

D Kidd, J Nelson, I Wallace, H Dusoir, S McKinstry, F Jones, V Patterson. Royal Victoria Hospital, Belfast, UK

Juvenile metachromatic leucodystrophy (JML) is a rare disorder of autosomal recessive inheritance, caused by deficiency of the lysosomal enzyme, aryl sulphatase-A. This allows accumulation of sulphatides in the nervous system and other organs. Although clinically heterogeneous within families, it is usually progressive and fatal.

We have previously reported a family with JML in which the index case developed symptoms age seven years and died at age 20. A biochemically affected sibling subsequently developed a progressive mental slowing at age 11 . A bone marrow transplant from a biochemically normal sister was performed, following which there has been no deterioration in the clinical examination, psychometric testing, and neuroradiological examination over a four-year period.

Bone marrow transplantation seems to have arrested the disease and should be considered a treatment option for these unfortunate patients.

INTRAFAMILIAL CORRELATION IN IDIOPATHIC TORSION DYSTONIA

NA Fletcher, AE Harding, CD Marsden. National Hospital for Neurology and Neurosurgery, London, UK

Idiopathic torsion dystonia (ITD) is a common inherited neurological disorder. Over
$85 \%$ of cases, regardless of severity or ethnic group, are caused by an autosomal dominant gene with $40 \%$ penetrance and highly variable expression. The phenotype varies from childhood onset generalised dystonia to adult onset focal dystonia or postural tremor. Severity is reliably predicted by age at onset.

Intrafamilial correlation coefficients using age of onset in pairs of affected relatives have been calculated in 100 ITD families. These are low (approximately 0.10 ) suggesting that the clinical features of ITD are due to environmental influences on ITD gene expression rather than the effects of other genes or genetic heterogeneity. The nature of these environmental factors is unclear but trauma may be one example and in some cases, iatrogenic injuries have been incriminated.

THE ROLE OF THE POSTERIOR LOBES FOR MOVEMENT CONTROL

H-J Freund. University of Dusseldorf, Dusseldorf, Germany

The significance of lesions of the frontal motor fields or their descending fibres are well recognised. In contrast, the impact of lesions posterior to the central sulcus for motor behaviour is less clear.

Whereas somatosensory deafferentation or the impairment of visuomotor projections causes pronounced tactile or visuomotor ataxia, damage of unimodal sensory association cortex not only produces perceptive and cognitive deficits but also gross motor dysfunctions. Evidence from the trajectory analysis of such deranged movements indicates that such motor disturbances are apractic in nature. They represent unimodal apractic deficits restricted to sensory guided motor behaviour but only for the affected modality. In contrast, lesions of polymodal association areas in the inferior parietal lobule of the language dominant hemisphere cause supramodal, conceptual apraxias such as ideational and ideomotor apraxia.

The results indicate that sensory association cortex is not only involved in the higher order processing of sensory information, but also in the elaboration of motor programmes.

AKINESIA IN PARKINSON'S DISEASE: A PET STUDY DEMONSTRATING IMPAIRED MESIAL FRONTAL ACTIVATION REVERSED BY APOMORPHINE

ED Playford, IH Jenkins, RE Passingham, W Fernandez, AJ Hughes, AJ Lees, RSJ Frackowiak, DJ Brooks. MRC Cyclotron Unit, Hammersmith Hospital, London, UK

Akinesia in Parkinson's disease may be related to abnormalities of the output from the basal ganglia to the frontal cortex. We performed two experiments measuring rCBF with PET to test this hypothesis. In the first we studied six patients with Parkinson's disease and six controls. Subjects were scanned at rest and while moving a joystick in a paced task in freely chosen directions. In the second six patients performed the same task both in the "off" state and when switched "on" with apomorphine. Significant increases in rCBF were determined with covariance analysis and Bonferroni statistics.

All subjects with Parkinson's disease 
performed the task successfully, but responded significantly slower than controls. Response time was significantly faster when "on" than "off". When "off" parkinsonian subjects activated motor and premotor cortex normally, but failed to significantly activate SMA and anterior cingulate. When "on" there was a significant improvement in SMA activation.

There is impaired activation of mesial frontal cortex in Parkinson's disease which is reversed by apomorphine. This may explain the difficulty parkinsonian patients have in initiating movements, and which is effectively treated with dopamine agonists.

CLINICAL AND IMAGING CORRELATIONS IN INHERTTED ATAXIAS

T Klockgether, U Wüllner, D Petersen, $\mathrm{J}$ Dichgans. University of Tübingen, Tübingen, Germany

The inherited ataxias comprise a wide spectrum of diseases with different clinical characteristics and heterogeneous neuropathology. Traditionally, classification of inherited ataxias was based on neuropathological criteria, but these classifications are of little relevance in clinical practice. In contrast, modern classifications are based solely on clinical, genealogical and aetiological criteria. Thus, Harding (1984) distinguished between inherited ataxias with known metabolic cause and the more common ataxias with unknown aetiology. The latter category comprises Friedreich's ataxia (FA), early onset cerebellar ataxias with special features (EOCA) and autosomaldominant cerebellar ataxia (ADCA). Recently, a late onset variant of FA (LOFA) has been described. In many cases of ataxia with an onset in adult life there is no evidence of inheritance and no symptomatic causes can be found. For these cases the term idiopathic cerebellar ataxia (IDCA) has been coined.

We have used MRI to study in vivo the macroscopical aspects of infratentorial and spinal cord pathology in 71 patients with different forms of ataxia. The aim of these examinations was to investigate which neuropathological categories correspond to the clinical and genealogical categories outlined above, and to see whether or not these categories are homogeneous with respect to atrophy changes observed in MRI.

All patients with FA and LOFA had spinal atrophy without major cerebellar involvement, whereas the MRI findings in EOCA were inhomogeneous (cerebellar cortical atrophy, olivopontocerebellar atrophy, spinal atrophy). All patients with ADCA coming from families with pure cerebellar symptomatology had atrophy changes confined to the cerebellum, whereas we observed different combinations of cerebellar, brainstem and spinal cord atrophy in patients with ADCA with additional non-cerebellar symptoms. Patients with ADCA fell into two different categories. Those with a lasting pure cerebellar syndrome had pure cerebellar atrophy, whereas all patients with additional non-cerebellar symptoms had olivopontocerebellar atrophy.

THE RELATIONSHIP BETWEEN NIGRAL PATHOLOGY AND ISOLATED TREMOR STUDIED WITH PET AND ${ }^{18} \mathrm{~F}$-DOPA DJ Brooks, ED Playford, V Ibanez, PD Thompson, LJ Findley, CD Marsden. MRC Cyclotron Unit, Hammersmith Hospital, London, UK
The relationship between essential tremor and Parkinson's disease is still uncertain. An increased incidence of essential tremor in relatives of patients with Parkinson's disease, and of patients with essential tremor developing Parkinson's disease, has been reported. Using ${ }^{18} \mathrm{~F}$-Dopa and PET we have studied the integrity of the nigrostriatal dopaminergic system in eight patients with familial essential tremor, 11 patients with sporadic, predominantly postural, tremor, and 10 patients with isolated, predominantly rest, tremor. Striatal ${ }^{18}$ F-Dopa influx constants $\left(K_{i}\right)$ were compared with those obtained for 30 age-matched controls, and 16 patients with L-dopa responsive Parkinson's disease. All eight familial tremor patients had normal individual striatal ${ }^{18} \mathrm{~F}$-Dopa uptake (mean putamen $\mathrm{K}$ 0.0085 (SD 0.0009), normal 0.0098L (SD $0.0011) \mathrm{min}^{-1}$ ). Two of the 11 subjects with sporadic postural tremor had reduced putamen ${ }^{18} \mathrm{~F}$-dopa $K_{\mathrm{i}}$ values, and both subsequently developed bradykinesia. All 10 patients with isolated rest tremor had reduced putamen ${ }^{18} \mathrm{~F}$-dopa uptake (mean putamen $\mathrm{K}_{\mathrm{i}} \quad 0.0050 \mathrm{~L}$ (SD 0.0013) Parkinson's disease 0.0047 (SD 0.0010 ) $\left.\mathrm{min}^{-1}\right)$. Our findings do not support an association between essential tremor and Parkinson's disease, but support the concept of a tremulous form of Parkinson's disease. The presence of cogwheel rigidity on synkinesis, reduced arm swing, and asymmetry of onset were not useful predictors of the presence of nigral pathology in patients with postural tremor.

DIAGNOSTIC AND THERAPEUTIC STRATEGIES IN HIV PATIENTS WITH ENCEPHALITIS W Enzensberger, PA Fischer. JW Goethe University, Frankfurt, Germany

Retrospective analysis (1983-91) of more than 600 HIV patients from Frankfurt (Germany) showed that more than $65 \%$ developed primary (directly HIV-induced) or secondary neurological problems (HIV $55.7 \%$, Toxoplasma gondii $20.7 \%$, CMV $8.9 \%$, Treponema pallidum $3.9 \%$, Cryptococcus neoformans $2 \cdot 6 \%$, JC-virus $2 \%$, HSV $0.3 \%$ ). Encephalitis was the most common manifestation of neurological involvement. Differential diagnosis is particularly difficult in HIV patients, as signs and symptoms are mostly nonspecific. Nevertheless, chronic cognitive decline, lack of focal neurological deficits, EEG slowing, and brain atrophy seen in CT or MRI scanning are typical findings in AIDS encephalopathy and should be treated with zidovudine. Patients with focal deficits suffer mainly from CNS toxoplasmosis or progressive multifocal leukoencephalopathy (PML). In CNS toxoplasmosis, hypodense lesions are seen in CT, which in contrast to PML are space occupying and contrast enhancing (similar to CNS lymphoma). If CNS toxoplasmosis is likely, empiric chemotherapy is indicated. If the initial CT scan is negative, HSV encephalitis should be suspected and treatment with acyclovir started. In CNS cryptococcosis CSF analysis is usually diagnostic. For the diagnosis of neurosyphilis antibody testing should be performed to justify empiric penicillin therapy.
MOLECULAR AND BIOCHEMICAL STUDIES OF A NEW MUTATION CAUSING LEBER'S OPTIC ATROPHY

LA Bindoff, $\mathrm{N}$ Howell, L Taylor, DM Turnbull. University of Newcastle upon Tyne, Newcastle upon Tyne, UK

Leber's hereditary optic neuropathy (LHON) is a significant cause of acute/ subacute visual loss that particularly affects young men. Although primarily a disease of the optic nerve, the condition may also be associated with other neurological (encephalopathy, deafness) and/or cardiac dysfunction (conduction defect). Genetic analysis has shown the disease is inherited by maternal transmission which suggests a mutation of mitochondrial DNA as this is itself inherited only from the mother. Recent studies have found a point mutation in the ND4 gene (at position 11 778) which encodes a subunit of the respiratory chain complex-NADH: ubiquinone oxidoreductase (complex I) (Wallace et al, Science $1988 ; 242: 1427)$. However, this mutation is only present in around $50 \%$ of the families studied. We have studied several families with LHON and in about $50 \%$ (that do not carry the ND4 mutation) have found a homoplasmic mutation involving another mitochondrially encoded subunit of Complex I-NDI. It is this subunit that is thought to contain the ubiquinone binding (catalytic) site. Our studies show that the mutation would disrupt a highly conserved region of the protein and would, therefore, be expected to produce adverse effects. Biochemical studies of mitochondrial fractions prepared from blood platelets have shown that complex I activity is lowered in all individuals carrying the mutation. Thus, unlike almost all previous studies, we have demonstrated the expected biochemical deficit in patients carrying a mutation of the mitochondrial genome. The molecular defect may be the cause of LHON in as many as $25 \%$ of affected individuals and its presence allows us to speculate about the relationship between mutations of mitochondrial DNA and clinical disease.

CENTRAL MOTOR CONDUCTION IN

DEGENERATIVE DISORDERS OF THE MOTOR SYSTEM

D Claus, B Neundörfer. University of Erlangen-Nürnberg, Erlangen, Germany

Central motor conduction (CMC) to abductor digiti minimi (ADM) and tibialis anterior (TA) was investigated after magnetic brain stimulation in 75 patients with degenerative disorders of the motor system. Of the 25 patients with spinal muscular atrophy (SMA) eight had Kugelberg Welander's and six Vulpian Bernhardt's disease. Fourteen patients had the pure form of hereditary spastic paraparesis (HSP). Motor neuron disease (MND) was diagnosed in 36.

In Kugelberg Welander's CMC to ADM was normal in all patients confirming a purely peripheral motor disorder. The scapulo humeral manifestation of SMA is known to often convert into MND. Four out of six patients had abnormal CMC, mainly amplitude reduction, results were asymmetric (5/11 sites abnormal). In HSP CMC was usually normal to upper extremities (13/14 patients) and abnormal to the legs (eight of nine patients). This is thought 
to be due to an emphasis of the degenerative process in the thoracic cord. In MND abnormal CMC to ADM was seen in 15/36 patients (19/70 sites) CMC to TA was abnormal in $11 / 20$ patients (14/40 sites). From these results it cannot be confirmed that CMC is a most sensitive test in early MND.

INTRACAVERNOSAL PAPAVERINE INJECTIONS FOR MEN WITH MULTIPLE SCLEROSIS AND ERECTILE DIFFICULTIES

CD Betts, SJ Jones, CJ Fowler. The National Hospital for Neurology \& Neurosurgery, London, UK

Impotence is common in men with multiple sclerosis but its neurological basis remains uncertain. We have studied 43 impotent patients with multiple sclerosis by neurophysiological and urodynamic tests. The feasability of treating these patients with intracavernosal injections administered from a neurological hospital has been assessed.

The mean age was 42.3 years and the mean duration of erectile difficulties was 3.5 years (range 1-11 years). Forty-one patients had urinary symptoms and half of these had developed erectile difficulties at the onset of their urinary symptoms. Thirteen patients were wheelchair bound

Pudendal evoked potentials were absent or delayed in 36 patients. Detrusor hyperreflexia and dyssynergia were the predominant urodynamic findings. Fifteen patients have used papaverine successfully for 12-18 months and 14 for 2-12 months. Only patients over the age of 50 required doses greater than $40 \mathrm{mg}$. Half of the patients have reported ejaculatory problems. Prolonged erection was the commonest complication but this was always easily reversible.

The study has demonstrated that intracavernosal injection therapy is a worthwhile treatment for patients with multiple sclerosis and even men with severe disability can benefit from this technique.

LOCALISED PROTON MAGNETIC RESONANCE SPECTROSCOPY IN METABOLIC AND

INFLAMMATORY ENCEPHALOPATHIES OF CHILDHOOD

E Wilichowski, F Hanefeld, HJ Christen, B Kruse, H Bruhn, J Frahm, HJ Bauer. University of Göttingen, Göttingen, Germany

Encephalopathies of childhood represent a heterogenous group of diseases with respect to aetiology, clinical course, diagnostic and therapeutical modalities.

Localised proton magnetic resonance spectroscopy (MRS), a non-invasive method allows estimation of specific cerebral chemical metabolites and may give more insight into the aetiology of these diseases.

MRS follow-up examinations in patients could reveal more precise information about the course of the disease and/or effects of treatment.

We report the results of combined MRI and localised proton MRS in 25 children with encephalopathies of heterogenous aetiology.

The following cases have been studied: subacute encephalomyelopathy (Leigh's disease): four patients; adrenoleuco- dystrophy: eight patients; leucodystrophy of unknown origin: three cases; Kearns-Sayresyndrome: one patient; MERRF: one patient; childhood multiple sclerosis: eight cases. The MRS findings are discussed in relation to MRI, clinical and laboratory data.

CHEMICAL AUTOREGULATION IS THE MOST POWERFUL DETERMINANT OF CEREBRAL BLOOD FLOW IN HEAD INTURED CHIIDREN PM Sharples, JA Eyre, D Matthews, PJ Crawford, AD Mendelow, I Chambers. University of Newcastle upon Tyne, Newcastle upon Tyne, UK

Provision of sufficient blood to meet the metabolic needs of the brain is the major aim of neurointensive care. Cerebrovascular resistance (CVR) is normally the key factor in the control of cerebral blood flow (CBF), but cerebrovascular responsiveness is often assumed to be impaired following head injury.

To investigate this assumption, we have measured CSF (Kety Schmidt method), cerebral perfusion pressure (CPP), cerebral metabolic rate $\left(\mathrm{CMRO}_{2}\right)$, arterial oxygen content, $\mathrm{paCO}_{2}$ and cerebrovenous $\mathrm{pH}$, base excess and lactate concentrations in 20 severely head injured children. CVR was calculated from CPP/CBF.

A total of 115 measurements were made in 20 children aged 2-16 years. CVR varied markedly between and within patients (range $0.2-2.8 \mathrm{mmHg} / \mathrm{ml}$ ). There was no consistent relationship between CVR and time after injury. $\mathrm{CMRO}_{2}$, arterial oxygen content, $\mathrm{paCO}_{2}$ and cerebrovenous acid base status were powerful determinants of CVR and CBF $(p<0.0001, p<0.01$, $\mathrm{p}<0.01, \mathrm{p}<0.02$, respectively), indicating that chemical autoregulation of CBF is preserved. CPP also emerged as an important determinant of CVR ( $<<0.001)$, suggesting preservation of pressure autoregulation.

In conclusion, the preservation of chemical autoregulation provides powerful mechanisms to alter CBF in children receiving intensive care. The variability of CVR and the evidence of preservation of pressure autoregulation indicates that CPP may not provide a reliable measure of $\mathrm{CBF}$.

APPARENT DECLINE OF ALS IN THE HIGHINCIDENCE DISEASE EPICENTER OF IRIAN JAYA AC Ludolph, PS Spencer. Oregon Health Sciences University, Portland, USA

Amyotrophic lateral sclerosis (ALS) and parkinsonism dementia reportedly have been phenominally frequent in three western Pacific populations: Chamorros of Guam and Rota (Mariana Islands) Japanese residents of Kii Peninsula (Honshu Island) and the Auyu linguistic group of Irian Jaya, Indonesia. The latter are hunter-gatherers who were settled in riverine villages constructed between 1937 and 1951. Field surveys $(1987,1990)$ of the largely sessile Auyu population (approximately 1200 subjects) of the River Ia suggest ALS prevalance has declined relative to that previously reported. Comparable trends have been noted in Guam and the Kii Peninsula. These changing disease patterns have occurred as the affected populations have undergone differing degrees of modernisation, including a decreasing use of the neurotoxic cycad seed for food
(Guam) and/or medicine (all three foci). In the lrian Jaya focus, this has occurred during a period of continued use of the same source of drinking (river) water, a suggested aetiological factor. The clinical picture of the Auyu extrapyramidal disorder consisted of bradykinesia, rigidity, and upper motor neuron signs.

COMPUTER SIMULATION OF NEURONE DEGENERATION AND FIBRE DENERVATION IN MOTOR NEURONE DISEASE

G Küther, H-G Lipinski. Technical University and MEDIS Institute, Munich, Germany

The temporal development of muscle fibre denervation and strength decline in motor neuron disease (MND) reflect basic properties of the underlying degenerative process. However, due to the presence of reinnervation, clinical and EMG data provide only indirect evidence for the loss of neurons. Several factors (ie time course of neuron death, sprouting rate, motor unit size) influence the efficiency of reinnervation and thus the clinical course of the disease. To analyse the complex interdependence of these factors, a computer model has been created which allows a simulation of the degenerative process with a free variation of each factor. The calculations showed that no proportionality existed between the number of denervated muscle fibres and the number of lost neurons. More than $50 \%$ of all neurons may be degenerated, before persistent fibre denervation becomes detectable. Efficiency of reinnervation not only depended on the sprouting rate, but also on the time course of neuron loss and the stage of degeneration. Based on these calculations, the time course of neuron decay can be deduced from available clinical and EMG data. The discrepancies observed between neuron loss and fibre denervation allow clinical limits to be defined, beyond which no therapeutic effects are detectable despite the efficiency of administered agents. Hence, the results are of interest for the planning and interpretation of therapeutic trials in MND.

SUBGROUPS OF ACQUIRED CHRONIC DEMYELINATING NEUROPATHY AND THEIR RESPONSE TO IMMUNOSUPPRESSANT DRUGS: STEROIDS INDUCE DETERIORATION IN A MOTOR SUBGROUP

M Donaghy. University of Oxford, Oxford, UK

The response to immunosuppressant drugs has been assessed in four patients aged 34 75 years, with purely motor forms of acquired chronic demyelinating neuropathy. They have been compared with the responses of 12 patients with sensorimotor chronic inflammatory demyelinating polyneuropathy (CIDP) and five with paraproteinaemic sensorimotor demyelinating neuropathy (PPDN). All patients were treated with prednisolone $60 \mathrm{mg}$ daily, converting to $45 \mathrm{mg}$ on alternative days over three to four months, and usually accompanied by azathioprine $2.5 \mathrm{mg} / \mathrm{kg} /$ day. Four additional patients were not treated (three PPDN, one CIDP with coexistent diabetes mellitus). Of the 12 patients with CIDP, 10 made moderate to excellent functional improvements, and two stabilised or improved only slightly. Of five with PPDN, 
all stabilised or improved moderately. All four patients with purely motor demyelinating neuropathy deteriorated significantly in the weeks following initiation of steroid therapy. Three lost the ability to walk, and one lost essential hand functions. Two of them obtained modest benefit from plasma exchange lasting a few weeks. One regained his previous state when steroids were stopped. Another returned to his usual state when steroids were stopped and cyclophosphamide and regular plasma exchange instituted. Two patients died, one as a result of the neurological deterioration, and one with infective complications after improving slightly with cyclophosphamide therapy. Steroids should only be used with extreme caution in patients with purely motor demyelinating neuropathy. The optimal form of therapy for motor demyelinating neuropathy awaits identification.

IMMUNOCYTOCHEMICAL STUDIES ON HUMAN NERVE BIOPSIES

B Schmidt, HP Hartung, KV Toyka. University of Würzburg, Würzburg, Germany

We have recently characterised the sequential expression of immune activation markers using immunocytochemistry in EAN. Prompted by these findings we have initiated an immunocytochemical study on sural nerve biopsies from patients with neuropathies of diverse aetiology.

Biopsy specimens were stained for $\mathrm{T}$ cells (UCHL 1), monocytes/macrophages (CD 68), IgG/IgM, terminal complement complex (TCC, C5b-9) and for ICAM-1 expression. Immunocytochemistry was performed on unfixed $6 \mu \mathrm{m}$ thick cryosections, for a more detailed analysis on paraformaldehyde fixed $1 \mu \mathrm{m}$ thin cryosections, and on paraffin embedded material. Two cases of mononeuritis multiplex, two cases of chronic inflammatory demyelinating polyneuropathy (CIDP) and one case of Guillain-Barré syndrome (GBS) were compared with three non-inflammatory cases (HMSN type II, systemic amyloidosis, tomaculous neuropathy).

Immunohistochemically, $\mathrm{T}$ cells and macrophages infiltrating vessel walls and perivascular space suggested a vasculitic disorder in the cases with the multiple mononeuropathy. In CIDP, GBS, and noninflammatory polyneuropathies $T$ cells and macrophages were present in very small numbers surrounding epineural blood vessels or were totally absent. CD 68 positive cells were rarely found endoneurally. Endoneural IgG/IgM staining indicating disturbance of blood nerve barrier varied in intensity being lowest in HMSN type II. In the GBS biopsy marked staining for IgM surrounding small endoneural and epineural blood vessels could be seen. A granular staining pattern of TCC was observed in the perineurium of all cases. In one case of CIDP there was a strong staining of vessel walls. In GBS an intravascular deposition of TCC was observed. ICAM-1 expression was found on the endothelial surface of blood vessels with varying intensity in all cases investigated. Infiltrating $\mathbf{T}$ cells also appeared positive.

Further studies in a larger number of biopsies are currently underway to characterise by means of immunocytochemistry local immune activation in neuropathies of diverse aetiology. This may yield insight into mechanisms of peripheral nervous system damage in these disorders.

GUILLAIN-BARRÉ SYNDROME: STUDIES ON CIRCULATING CYTOKINES

HP Hartung, B Schmidt, KV Toyka. University of Würzburg, Würzburg, Germany

Immunoinflammatory responses are orchestrated by cytokines. We determined circulating levels of IL- $1 \beta$, IL-6, TNF- $\alpha$, and neopterin (a measure of IFN- $\gamma$ activity) in Guillain-Barré syndrome (GBS), a neuropathy of presumed immunopathogenesis. Serum/plasma samples were collected from 39 patients with GBS within five days of disease onset. Control samples were collected from patients with metabolic neuropathies, patients with other noninflammatory neurological disorders (OND), and healthy individuals. Cytokine concentrations were quantitated by sensitive radioimmunoassays. $44 \%$ of patients with GBS had increased levels of neopterin, a macrophage product released upon IFN- $\gamma$ stimulation, $40 \%$ had elevated levels of IL-6, but only 8 and $5 \%$ had measureable concentrations of IL- $1 \beta$ and TNF- $\alpha$ respectively. Serial measurements revealed a decline of increased circulating levels of neopterin and IL-6 that paralleled clinical improvement. The presence of neopterin temporarily related to disease activity reflects macrophage activation in GBS and emphasises a pathogenic role for IFN- $\gamma$ in immune-mediated peripheral nervous system damage. On the other hand, elevated IL-6 levels may mirror B cell activation in GBS.

\section{Poster presentations}

ELEMENTARY MECHANISMS OF PERIODIC EPILEPTIFORM DISCHARGES

OW Witte, T Dorn, $R$ Domann. Neurologische Klinik, Heinrich Heine University, Düsseldorf, Germany

Periodic epileptiform discharges are the hallmark of a variety of focal and diffuse cerebral disturbances. They occur in herpes encephalitis, Jakob-Creutzfeldt disease, subacute sclerosing panencephalitis, following anoxia and in metabolic encephalopathy. Some of these and other conditions may be differentiated by different intervals and patterns of the periodic discharges. The aim of the present study was to analyse mechanisms causing such periodic discharges.

Periodic epileptiform discharges could be induced by focal application of penicillin to the rat cortex. In the EEG spikes occurred in different rhythms which were labelled irregular, composed and regular and had mean interval lengths between successive spikes of about 2000, 1000, and $300 \mathrm{~ms}$, respectively. Concomitantly with the EEG spikes neurons within the focus displayed paroxysmal depolarisation shifts (PDS). In $70 \%$ of the recordings the PDS were followed by inhibitory afterpotentials which lasted as long as the interval between successive discharges. In these neurons the PDS arose from a hyperpolarised baseline level. In areas surrounding the focus the cells were inhibited concomitantly with
EEG spike. These inhibitions never lasted longer than $1000 \mathrm{~ms}$. Averaging of recordings from such neurons with respect to the EEG spike revealed that in about $20 \%$ of the recordings a depolarisation preceded the inhibition by up to $400 \mathrm{~ms}$.

The investigations showed that different areas within and around epileptiform foci can be involved in generating periodic epileptiform spikes. The pacemaker activity can be located outside the focus itself.

TYPICAL ABSENCE SEIZURES IN ADULTS: I. CLINICAL-EEG AND VIDEO-EEG FINDINGS IN 23 PATIENTS

CP Panayiotopoulos, E Chroni, C Daskalopoulos, A Baker, S Rowlinson. St. Thomas' Hospital, London, UK

We report combined clinical, EEG and video-EEG findings of 23 adult patients (age range $=21-67$ years, mean $(S D)=$ $35.9(11 \cdot 3)$ ) with typical absence seizures. There was a $10 \%$ prevalence amongst random clinical referrals of adult patients with epileptic seizures with a female predominance (18 women). Clinically, the absences showed considerable quantitative and qualitative variation from mild and difficult to detect to those with severe impairment of consciousness. They were documented with EEG and video-EEG studies. Onset of absences ranged from 7-46 (mean 18.3 $(10 \cdot 5)$ ) years of age; six patients had onset of absences after the age of 20 years. Generalised tonic-clonic seizures occurred in 20 patients; their frequency varied from rare (one or a few in life time) to as many as one per week. Myoclonic jerks of the limbs (11 patients) were not usually associated with the absence ictus as opposed to the eyelid (five patients) and perioral (two patients) myoclonus which was consistently linked with the absence ictal manifestations. Absence status occurred in five patients and was recorded with EEG or video-EEG in four of them. Absence seizures were frequently unrecognised for years and in eight of them the diagnosis of complex partial seizures was prevailing. The most commonly used drug was carbamazepine with 10 patients on polypharmacy. Satisfactory control of the seizures was achieved with sodium valproate. The EEG, particularly video-EEG, is invaluable in diagnosis but focal abnormalities (seven patients) may be erroneously interpreted as indicative of partial seizures. Video-EEG recordings from patients with juvenile, eyelid, symptomatic and other absences will be shown to document our findings.

TYPICAL ABSENCE SEIZURES IN ADULTS: II. SYNDROMIC CLASSIFICATION

CP Panayiotopoulos. St. Thomas' Hospital, London, UK

An epilepsy disease syndrome diagnosis of 23 adult patients with absences was attempted in accordance with the Proposal of the International League Against Epilepsy (ILAE). Juvenile myoclonic epilepsy with the triad of idiopathic generalised seizures which are age-related, the circadian distribution of the myoclonic jerks on awakening and the characteristic clinico-EEG manifestations of the typical absences was easy to recognise in seven $(30.4 \%)$ patients. Four patients $(17 \cdot 4 \%)$ were classified as 
juvenile absence epilepsy on the basis of multiple clinico-EEG data (age at onset, combined ictal clinico-EEG manifestations, frequency of the seizures, absence of myoclonic jerks on awakening, absence of ictal eyelid myoclonia or symmetrical myoclonic jerking or early onset GTCS). Ictal eyelid myoclonia with absence seizures was consistently recorded in three patients $(13 \%)$; it was also occasionally seen in one patient with symptomatic absences and in another patient during myoclonic status. Only one of the three patients would meet the criteria of the syndrome described by Jeavons, i.e. photosensitive patients with absences that are resistant to treatment and show marked jerking of the eyelids associated with spike wave-discharges on eye-closure. There was no uncertainty in classifying three other patients as late onset absence status, photo/pattern sensitive epilepsy with absences and symptomatic epilepsy with typical absences, respectively. The remaining six patients $(26 \%)$ could not be classified with confidence; analysis of their clinico-EEG profile and the discussion of their differential syndromic diagnosis indicates that there maybe more epileptic syndromes with absences than presently recognised, i.e. perioral myoclonic absence epilepsy. It is concluded that despite well known drawbacks of the proposed ILAE classification, a disease/syndrome diagnosis is possible.

EARLY DEMONSTRATION OF AXONAL NERVE LESIONS BY ELECTRONEUROGRAPHY

T Wißmeyer, P-J Hülser. Abteilung Neurochirurgie, Ulm, Germany

Pathologic spontaneous activity (SA) is a reliable but delayed sign of axonal nerve lesions. We studied the diagnostic value of the amplitude of the evoked compound muscle action potential (CMAP) after distal stimulation of the injured nerve. In $95 \%$ of 54 healthy persons the differences between the right and left side after stimulation of the median, ulna, peroneal, and tibia nerve did not exceed $30 \%, 30 \%, 32 \%$, and $50 \%$ respectively. In another group of 30 healthy persons the effect of positioning the different electrode on the M.abductor hallucis has been examined and the difference between the sides comparing the maximum amplitude of five different positions on each side did not exceed $30 \%$. In 25 patients with unilateral acute mechanical nerve lesions the amplitudes of the CMAP on both sides were measured within a few days (75\% 3-7) after the acute event (at this time only two cases of SA). In 24 cases the difference in the amplitudes were obviously in the pathologic range. We demonstrate that reduction of the CMAP after distal stimulation of the injured nerve precedes the occurrence of SA and provides a high degree of sensitivity especially by means of using multi-channel recording and taking care of the electrode position over the muscle belly.

COGNTTIVE EVENT RELATED POTENTIALS AND TEMPORAL LOBE DYSFUNCTION

RC Roberts, MD Rugg, DD Potter, CD Pickles, ME Nagy. University of Dundee and University of St. Andrews, UK

Event related potentials (ERPs) are small perturbations in the EEG, which are revealed by averaging the EEG after a repetitive event or task. It is generally held that early components are related to the sensory input and later components to the cognitive processing required by the task We have used a recognition memory task in which words are presented visually and the subject must decide whether each word is being presented for the first or second time. Second presentations occur after six intervening items. In a control group $(n=15)$ with idiopathic generalised epilepsy, ERPs to correctly recognised repeated words were more positive-going in the $300-600 \mathrm{~ms}$ post-stimulus latency range, than ERPs to the first presentation of the words. This difference was significantly smaller in subjects who had had a left $(n=8)$ or right $(n=8)$ temporal lobectomy, and did not differ reliably from zero. In $20 \%$ of temporal lobe epilepsy patients with left $(n=14)$ or right $(\mathrm{n}=11)$ foci this difference was also abnormally small $(>2$ SD below control mean), and these groups were characterised by asymmetrical ERP differences, which were smaller over the hemisphere ipsilateral to the focus. This and other similar ERP procedures might provide non-invasive means of investigating medial temporal lobe dysfunction.

INTRAVENOUS AND INTRATHECAL THERAPY WITH INTERFERON-BETA IN AETIOLOGICALLY UNCLARIFIED ENCEPHALITIS

KP Westphal, PJ Hülser, KH Wollinsky, ME Westarp, J Bauer, $\mathrm{H}$ von Eick, $\mathrm{HH}$ Kornhuber. University of Ulm, Ulm, Germany

Results of 14 patients who were treated with interferon- $\beta$ (IFN- $\beta$ ) (Rentschler, Laupheim, Germany) in encephalitis are reported. The clinical diagnosis had been proven by pleocytosis and increased protein in the CSF as well as by means of CCT and MRI. Two patients had HSV encephalitis. Nine out of the 14 patients had a severely changed consciousness or were comatose. Four patients had mild changes of consciousness but pronounced deficits and foci of liquefaction in the MRI, they were diagnosed as chronic encephalitis. One patient had Rasmussen's encephalitis. Except for patients with chronic encephalitis nearly all received acyclovir and a combination of antioedema therapy. In seven patients IFN- $\beta$ was administered only intravenously $\left(0.30-0.50 \times 10^{6} \mathrm{U} / \mathrm{kg}\right)$, and three patients were treated intrathecally about 10 times with $10^{6} \mathrm{U}$ for five weeks. Four patients received a combination. Out of the nine patients with severe changes of consciousness, in one only an improvement of the oedema was found, five patients were cured with some deficits, three patients were without deficits after treatment. The patient with Rasmussen's encephalitis had an improvement of the EEG. Three patients with chronic encephalitis had a clear improvement of the neurological deficits, and one had a complete remission. Drug fever higher than $40^{\circ} \mathrm{C}$, leucopenia, thrombocytopenia, increase of PTT, and decrease of fibrinogen were only observed during intravenous treatment. Lower costs and less side effects suggest a role for the intrathecal application of IFN- $\beta$ in encephalitis.
OUTCOME OF AGGRESSIVELY TREATED MALIGNANT MENINGITIS

R Grant, HS Greenberg, L Junck University of Edinburgh, Edinburgh, UK and University of Michigan, Ann Arbor, Michigan, USA

Malignant meningitis usually occurs as a late neurological manifestation of cancer, and even with aggresive treatment, surviva is usually less than four months. We reviewed the case records of 36 consecutive patients with cytologically proven malignan meningitis to try to identify factors which led to an improved outcome.

Twenty-eight patients had adenocarcinoma, five had small cell lung cancer and three had melanoma. Thirty-three patients were actively treated (29 intrathecal chemotherapy, 18 radiation therapy (RT), 12 intravenous chemotherapy) and three refused treatment. Similar numbers of patients presented with cerebral, cranial nerve, and spinal nerve symptoms.

Median survival for treated patients was only eight weeks. Twelve per cent had improvement in symptoms and $33 \%$ lived for more than four months. Six patients with breast carcinoma who were treated with intrathecal chemotherapy ( \pm RT) had a median survival of three weeks (range 2-11 weeks) while seven patients treated with intravenous and intrathecal chemotherapy ( \pm RT) survived 20 weeks (range 5-31 weeks). Two of 23 intraventricular reservoir replacements were complicated by intracranial bleeding and three required repositioning.

There was no relationship between site of involvement and treatment response. Median survival is poor despite aggressive treatment. Intravenous chemotherapy may improve survival and deserves further study.

MANAGEMENT OF MENINGOENCEPHALITIS IN NON-HIV IMMUNODEFICIENCY

M Strittmatter, G Hamann, V Jost, M Stoll, $\mathrm{K}$ Schimrigk. University of Saarland, Homburg, Germany

Immunodeficient patients are at high risk of acquiring a severe meningoencephalitis, especially patients with haematological, oncological diseases and long-term alcoholism.

A total of 37 patients with severe meningoencephalitis were retrospectively studied. Sixteen patients (group 1) had a history of such a non-HIV immunodeficient state and 21 patients (group 2) had no signs of any immunodeficiency.

Despite similar initial state (concerning consciousness disturbances, focal-neurological deficits, number of CSF-cells, CSF-protein) the outcome of group 1 was significantly worse $(p<0.01)$. Especially the 12 patients in group 1 with bacterial meningoencephalitis showed a bad prognosis with a mortality of $50 \%$. Therapeutic intervention was much more extensive in immunodeficient patients using three or fourfold antibiotic combinations and immunoglobulins. In patients with no former immunodeficiency the antibiotic combination of a cephalosporin (third generation) and aminoglycoside was able to reduce inflammatory signs in all cases. Complications of the cerebrovascular microcirculation caused by reactively increased plasma fibrinogen and viscosity were only seen in group 1 . 
We prefer an initial combination of an aminoglycoside and a cephalosporin of third generation even in immunodeficient patients. Moreover the microbiological identification succeeded only in $28.9 \%$ of both groups. The routine expansion of therapeutic management in meningoencephalitis does not improve the outcome in immunodeficient patients.

SACCADIC EYE MOVEMENTS ARE IMPAIRED BY TRANSCRANIAL MAGNETIC STIMULATION (TMS)

WH Zangemeister, G Beckers, A Canavan, V Hoemberg. University of Hamburg, Hamburg and University of Düsseldorf, Düsseldorf, Germany

Transcranial magnetic stimulation (TMS) of occipital, parietal, central and frontal cortex was administered in five human subjects while performing a visually cued alternating saccade task and during fixation of a central stimulus. TMS failed to induce eye movements. Saccadic trajectories could however be changed or saccades could even be abolished when stimulation was administered within $100 \mathrm{~ms}$ before saccade onset. This effect was particularly marked with TMS of the frontal cortex. TMS did not affect saccades when administered after they had started. It is concluded that TMS interferes with saccadic pre-programming.

ANALYSIS OF HORIZONTAL SACCADES IN MULTIPLE SCLEROSIS IS A USEFUL CLINICAL TOOL

I Ritouret, $\mathrm{AD}$ Collins, JM Gibson. Royal Victoria Hospital, Belfast, UK

Horizontal saccades can be measured accurately and should provide information about brainstem function. Previous studies have failed to provide results robust enough for routine clinical use. We have attempted to overcome this problem by using only data from small saccades, separated by eye and direction ( $a b$, or adduction), and analysed using simple mathematical models. Infrared recordings of saccades were made from 50 normal subjects (18-71 years, mean 40 ), and 53 patients with multiple sclerosis (20-66 years, mean 48 ). Targets were nine diodes within $a \pm 10^{\circ}$ range, and both monocular and binocular recordings were made. VER and BAEP were also recorded. All patients were clinically definite or probable (Poser) and were divided into three groups on the basis of ocular motor signs or history. (Group A: clinically abnormal eye movements; Group B: positive history of, but no current clinical eye movement signs; Group C: neither past history nor current signs.) Results falling beyond 3 SD from the mean were considered abnormal. Duration proved a more useful parameter than peak velocity. Incidence of abnormalities was eye movements: A $-100 \%$, B - $60 \%$, C - 43\%; VER: A - 79\%, B $67 \%, \mathrm{C}-57 \%$; BAEP: A - 30\%, B - 33\%, $\mathrm{C}-37 \%$. By segregating our results we can suggest specific anatomical lesions underlying a particular set of data. This method provides clinically useful data about functions commonly affected in multiple sclerosis.
THE CORTICOSPINAL TRACT IN STROKE: AN INVESTIGATION WITH TRANSCRANIAL

MAGNETIC STIMULATION

M Schubert, KR Mills, SJ Boniface, R Dengler. University of Bonn, Bonn, Germany and University of Oxford, Oxford, UK

Corticospinal tract functions can be investigated with transcranial magnetic stimulation. In conditions with upper motor neuron lesions abnormal compound muscle action potentials following transcranial stimulation are observed. The pathophysiology of these abnormalities was studied in single motor unit responses of the first dorsal interosseous muscle. The first period of increased firing probability in peristimulus time histograms (100 to 500 magnetic stimuli) was analysed in 15 motor units (MUs) of eight patients with stroke and $26 \mathrm{MUs}$ of 14 healthy controls. MUs of controls showed excitory responses at 17 to $30 \mathrm{~ms}$ after the stimulus. In five MUs of patients responses were normal. In five MUs there was no excitatory response. In five MUs responses with increased latencies and durations and bi- or trimodal peaks were seen. Intermodal intervals ranged from 4 to $18 \mathrm{~ms}$. The results suggest dispersion of conduction velocities or conduction block in corticospinal axons or activation of other descending pathways.

TRANSCALLOSAL INHIBITION OF THE MOTOR CORTEX IN PATIENTS WITH HEMIPLEGIA OF VASCULAR ORIGIN

A Ferbert, B Boroojerdi, K Diefenbach, H Buchner. RWTH, Aachen, Germany

If one stimulates the motor cortex with a figure of eight-shaped magnetic coil, an inhibition of the ongoing EMG activity can be observed in small hand muscles ipsilaterally to the stimulated hemisphere. We investigated 10 patients with vascular lesions leading to dense hemiparesis with paralysis of the hand. The motor cortex of the affected hemisphere was stimulated with an eight-shaped magnetic coil 6 to $7 \mathrm{~cm}$ lateral to $\mathrm{Cz}$. Patients were asked to activate their nonparetic first dorsal interosseus muscle (FDI) ipsilateral to the stimulated hemisphere. The EMG was rectified and averaged. None of the patients showed a direct response in the contralateral FDI. In the ipsilateral (nonparetic) FDI an inhibition of the ongoing EMG activity was observed in all four patients with pure subcortical infarction with an onset latency of 30-35 ms. Three of the six patients with cortical-subcortical infarction showed no inhibition and the latter was delayed in two of the remaining patients.

As inhibition was preserved in subcortical lesions destroying the pyramidal tract we conclude that this inhibition is not mediated through an ipsilateral projection but via a transcallosal route. It might have a bearing on the prognosis of hemiplegia whether transcallosal connections are affected by the lesion as well.

\section{DETECTION AND QUANTIFICATION OF}

PROVIRAL HIV DNA IN CSF

H Steuler, S Munzinger, B StorchHagenlocher, B Wildemann. University of Heidelberg, Heidelberg, Germany

Infection with human immunodeficiency virus (HIV) is frequently associated with neurologic complications. These may be due to opportunistic infections or may directly result from HIV infection of the nervous system. It is not known if the development of neurologic dysfunctions is related to the cellular infection rate in the CNS.

We determined the number of proviral copies in the CSF of 12 patients in various stages of HIV infection using serial dilutions of cell lysates from CSF which were subjected to a double polymerase chain reaction.

In all samples proviral DNA was clearly detected. The cellular infection rate ranged from one provirus per 25 cells up to one per 4800 cells with most of the samples falling into the range one per 25 to 500 cells. Interestingly this is a much higher proportion of infected cells than is reported for blood mononuclear cells.

DIAGNOSIS OF CYTOMEGALOVIRUS ENCEPHOLOMYELORADICULITIS BY DEMONSTRATION OF VIRAL ANTIGEN IN CSF CELLS

E Stark, J Haas. Hannover Medical School, Hannover, Germany

Cytomegalovirus infection is one of the most common opportunistic infections in patients with acquired immune deficiency syndrome. The incidence of infections of the nervous system is still unknown, although some distinct clinical syndromes have been described. As most diagnostic procedures such as viral culture and serological demonstration of a specific immune response are not very effective in this disorder, most cases of these infections have been diagnosed by post mortem examination. CSF cells were prepared by a special cytospin technique. Immunostaining for CMV immediate early antigens was performed by a double bridge enzyme ant enzyme procedure with monoclonal antibodies. In a series of 84 CSF samples from 69 HIV infected persons we have only found CMV bearing cells in three samples. All three patients had clinical syndrome compatible with a CMV infection of the nervous system (one brainstem encephalitis, two myeloradiculitis). Of the patients without CMV antigen no similar clinical pictures were observed. This result makes it probable that direct demonstration of viral antigens may be a solution to this diagnostic dilemma in a disease which is now treatable.

WHIPPLE'S DISEASE AS A RARE CAUSE OF ENCEPHALITIS

M Spranger, S Schwab, U MeydingLamade, R Schröder. University of Heidelberg, Heidelberg, and University of Cologne, Cologne, Germany

Central nervous involvement in Whipple's disease is a rare, but well known complication. We report here two cases of Whipple's disease confined to the CNS. The patients presented with a history of fever, apathy, arthralgia, loss of weight, and double vision. On admission both patients had complex disturbances of eye movements, facial paresis, hemiparesis, and hemiataxia. In both patients intestinal biopsy was negative. CSF investigations showed pleocytosis, in one case CSF cytology revealed PAS positive, sickle-shaped particles within macrophages. 
In the other case the diagnosis was confirmed by histology of the brain. Serial MRI in both patients showed multiple areas of gadolinium-enhancing lesions localised around and in the upper brainstem, compatible with abscesses or granuloma.

Whipple's disease should be considered in patients with unclarified encephalitis associated with complex gaze disturbances. MRI of the brain and PAS positive particles in CSF cytology may give clues, but the final diagnosis is made by biopsy of brain lesions. Both patients improved following treatment with chloramphenicol and long term administration of tetracyclines.

DIAGNOSIS AND THERAPY OF PERIVASCULAR LISTERIOSIS GRANULOMA IN THE ADULT H-J Reiss, W Büchele. Bezirkskrankenhaus Haar, Haar, Germany

Infections of the CNS with Listeria monocytogenes can offer diagnostic problems in the early stage of the disease. A case report is presented where the localisation of inflammatory lesions in the pontomedullary brainstem and adjacent cerebellum together with the findings of evoked responses and MRI led to the diagnosis which was confirmed by identification of bacterium in blood culture and which was the basis for successful treatment.

After preceding gastrointestinal symptoms a 57-year-old, hitherto healthy, woman suffered from fever up to $40.5^{\circ} \mathrm{C}$ and presented with rapidly progressing brainstem and cerebellar signs and symptoms without meningeal irritation. Acoustic evoked responses revealed brainstem dysfunction, CCT and MRI showed a perivascular granuloma on the floor of the IV ventricle with a surrounding oedema in the posterior fossa. CSF was inflammatory with lymphocellular pleocytosis of $900 / \mathrm{mm}^{3}$ and a total protein amount of $107 \mathrm{mg} / \mathrm{dl}$ Antibiotic therapy was instituted. During the first few days after admittance the status of the patient deteriorated due to the spreading oedema.

The therapy with ampicillin and gentomycin was continued and the patient improved gradually over several weeks, BAER and MRI were completely norma and the patient could be transferred to a rehabilitation unit.

Pontocerebellar signs and symptoms are the clinical hallmark of this infectious disease. MRI was found to be extremely sensitive in establishing the diagnosis which was later confirmed by microbiological and serological results.

PSEUDORABIES AS CLINICAL MANIFESTATION OF VIRAL BRAINSTEM ENCEPHALITIS

K Knafla, J Haas, H Kolbe, E Stark. Hannover Medical School, Hannover, Germany

A 41-year-old man suffered from rapidly progressive dysphagia, vomiting, persistent myoclonus of his diaphragm and left leg, sweating attacks and general weakness for three weeks. Neurological examination revealed horizontal ophthalmoplegia, dysasthesia of left face, oropharyngeal myoclonus, and disturbed swallowing. Tendon reflexes were brisk, Babinski sign bilaterally positive. Within three days myoclonus generalised, hydrophobia and psychotic disturbances occurred, and severe salivation made tracheal intubation necessary. First CSF yielded $114 / \mathrm{mm}^{3}$ cells (lymphocytes, plasmocytes). Two weeks later oligoclonal bands appeared and disappeared within four weeks. The second MRI after one month revealed white matter lesions in the parietel lobe, right brainstem and left basal ganglia. The clinical picture was typical of rabies. The patient had spent several years in China where urban rabies is endemic. Blood, CSF, urine, tracheal secretion, saliva and tear fluid were analysed for rabies virus several times including inoculation of animals, but remained negative, as well as results of $\mathrm{HSV}, \mathrm{CMV}$, tuberculosis, and Borrelia burgdorferi. An identical infectious bulbar paralysis, called MB Aujeszkyi, is described for animals being similar to rabies infection, and some case reports presumes this illness in humans. Antibiotics and acyclovir were without real improvement. In regard of acyclovir-resistant virus the new virostatic drug foscarnet was given for seven weeks. The patient recovered, only remaining with a dystonic right foot.

\section{PROGNOSIS IN NON-BACTERIAL}

\section{MENINGOENCEPHALITIS}

C Hansen, A Arlt, K Kunze. University of Hamburg, Hamburg, Germany

From 1984 to 1990, 60 patients with nonbacterial meningoencephalitis were treated in our hospital (28 men, mean age 33 years; 32 women, mean age 34 years). Admission was seven days on average after the start of symptoms. In 16 patients, a causal infectious agent could be identified (herpes simplex 7 , varicella zoster 3 , coxsackie 2 , adenovirus 2 , measles 1 , rabies 1 ). In the total group, $24 / 60$ patients had a good outcome with Karnofski index $(\mathrm{Ki}>=90$ $(0-1)), 18 / 60$ had a fair outcome $(\mathrm{Ki}>=70$ and $<90,(0-2)$ ), and 18/60 faired poorly $(\mathrm{Ki}<=60,(0-3))$. A favourable outcome was associated with adenovirus $(0-1$ in $2 / 2$ patients or coxsackie infections $(0-1$ in $2 / 2)$, but prognosis was worse for patients with herpes simplex $(0-3$ in $5 / 7)$ or varicella zoster $(0-3$ in $2 / 3)$ infections. Initial signs associated with poor prognosis included depression of consciousness $(0 / 24$ patients with $0-1,1 / 18$ with $0-24 / 18$ with $0-3$ ) focal neurological signs ( $2 / 24$ with $0-1,5 / 18$ with $0-2,6 / 18$ with $0-3)$, epileptic seizures (1/24 with $0-1,3 / 18$ with $0-2,7 / 18$ with $0-3)$, and an abnormal CT scan (1/11 with $0-1,6 / 15$ with $0-2,14 / 18$ with $0-3$ ). Initial CSF findings were not helpful for prognostic assessment. These findings will be related to the results of a larger local multicentre study on prognostic factors in nonbacterial meningoencephalitis (Hamburg Encephalitis Study) to support early therapeutic strategies.

\section{A LONGITUDINAL STUDY OF MAGNETIC} RESONANCE IMAGING (MRI) IN HIV INFECTION

H Manji, BE Kendall, AR Valentine, S Connolly, MJG Harrison. Middlesex Hospital, London, UK

The issue of whether HIV-infected asymptomatic individuals show evidence of neurological/neuropsychological dysfunction is still under study. As part of the multidisciplinary Middlesex/MRC Neurology HIV Cohort Study, we have used MRI to determine the prevalence of abnormalities in this asymptomatic group and to follow the natural history in an interval study.

Serial scans have been performed at a mean interval of 13 months on 72 homosexual/bisexual men (22 seronegative, 38 asymptomatic HIV seropositive, and 12 ARC/AIDS). Qualitative reporting of the scans was performed by two independent neuroradiologists blinded to all clinical details except age.

Cross-sectional analysis shows the prevalence of focal white matter lesions to be no more frequent in the asymptomatic group $(2 / 38)$ compared to the seronegative controls (7/22). No correlation between the prevalence of these lesions and age, alcohol intake, substance abuse, smoking or previous history of syphilis was found. There were no significant differences between groups in terms of cerebral atrophy. Assessment of change between the scans revealed no evidence of progression in the asymptomatic group. However, a significan number of ARC/AIDS subjects (4/12) showed an increase in cerebral atrophy as did 3/7 subjects who progressed from being asymptomatic to ARC/AIDS during the study period.

These results suggest that MRI shows no evidence of parenchymal brain damage in HIV-infected asymptomatic individuals over a period of 13 months. Results from other aspects of this study which include clinical neurological assessment, a battery of neurophysiological and neuropsychological tests concur with this finding. However, progression of cerebral atrophy on MRI may be found in some ARC/AIIS individuals including some progressors.

CHRONIC HERPES SIMPLEX ENCEPHALITIS IN A MAN WITH MYELOID LEUKAEMIA J Haas, K Knafla, E Stark, U Wurster, H Becker, GF Walter, H Poliwoda, H Hundeshagen, H Dietz. Hannover Medical School, Hannover, Germany

Herpes simplex encephalitis in patients with immunodeficiency syndrome is well known. In a 54-year-old man we first observed a severe leukaemic meningitis. After successful intrathecal treatment with methotrexate the patient recovered. Some weeks later he was admitted with headache, aphasia, hemiplegia, focal seizures and impaired consciousness. At the same time severe herpes genitalis occurred. Lumbar puncture now revealed xanthochromia $504 / \mathrm{mm}^{3}$ lymphocytes and elevated albumin. On CT, multilocular hypodense white matter lesions were seen. Acyclovir $10 \mathrm{mg} / \mathrm{kg} /$ day was given for six weeks. The patient recovered but there were mild neuropsychological disturbances. Five months later, repeat CT detected an increase of white matter lesions. In the CSF lymphocytosis persisted. On MRI, in addition, pachymeninges showed gadolinium enhancement. Aphasia became worse and a diagnostic biopsy of the meninges and parietal lobe revealed histologically mild chronic inflammation in the meninges. Gene-amplification from the pachymeninges revealed herpes simplex type II. In the follow-up there was concurrent reactivation of neurological signs and exacerbation of cutaneous herpes simplex. Our therapeutic efforts with repeated high dose of acyclovir, foscarnet, and immunoglobulins seem only to slow the progression of the disease. We suggest the chronic course 
of the herpes simplex type II meningoencephalitis is due to the extreme immunosuppression immediately before its acute manifestation.

CYCLOSPORIN A VERSUS AZATHIOPRINE IN MULTIPLE SCLEROSIS: SEVEN YEAR FOLLOWUP AND LONG-TERM SIDE EFFECTS

J Haas, E Stark, U Wurster, U Dommasch, L Kappos, S Poser. Hannover Medical School, Hannover, Germany

The German Multicentre Study Cyclosporin A (CIA) versus Azathioprine (AZA) in Multiple Sclerosis, finished in March 1986, and had revealed no differences in outcome after two years of treatment. In a long-term follow up we observed the course of the disease of the Hannover patients after the end of the trial. Every six months the severity of the disease in each patient was evaluated by rating scales: neurostatus, Kurtzke disability scale and incapacity scale, and ambulation index. The volumes of the MRI lesions in the forebrain hemispheres were determined by a three dimensional computer assisted planimetry. MRI lesion volumes at re-examination had diminished on both groups. Meanwhile, the patients with multiple sclerosis were followed-up for seven years. The therapeutic regimen in both groups was not different. In the AZA group $(n=34) 16$ patients were still on AZA, in the CIA group $(n=39)$, in 14 patients the CIA therapy was re-established after abrupt discontinuation in 1986 . In both groups the mean overall progression was minor, but in the CIA group 10 were wheelchair bound, versus 21 patients in the AZA group. The annual relapse rate decreased in the AZA group, but increased in the CIA group, due to a high number of exacerbations after the abrupt discontinuation of CIA. With the exception of mild hypertension the overall tolerence in the 14 re-treated CIA patients was satisfactory.

RELAPSE OF HERPES SIMPLEX ENCEPHALITIS: DIAGNOSTIC AND THERAPEUTIC STRATEGIES HT Eder, W Trabert, G Holzer. University of Saarland, Homburg, Germany

The introduction of the specific antiviral acyclovir led to a remarkable improvement in the treatment of encephalitis caused by herpes simplex (HSE). Although this agent has proved to be effective for treatment of HSE, especially in decreasing the mortality from $70 \%$ to $20 \%$, the disease may reoccur.

By presenting a case of recurrent HSE and reviewing the cases reported in the international medical literature, we discuss the possible pathophysiologic pathways and diagnostic difficulties in these rare variants.

The decision to begin treatment must be as early as possible. Because rapid diagnostic tests for HSE-infection are not available, treatment has to be decided upon on clinical criteria alone.

Treatment with acyclovir has to be continued for up to 21 days if there is no improvement in the clinical state, and focal neurological signs in particular. In cases of deterioration after the end of treatment, a relapse of HSE has to be assumed and the treatment should be restarted immediately.
OUTCOME AFTER RESUSCTTATION FOR VENTRICULAR FIBRILLATION

P Marx, H Mast for the FLUNA Study Group. Free University of Berlin, Berlin, Germany

In a prospective, randomised trial, 435 patients resuscitated for ventricular fibrillation received either the calcium antagonist flunarizine $(n=213)$ or placebo $(n=222)$ intravenously. We report here on the results of the placebo group only. Sixteen patients had to be excluded for protocol violations. Follow up was incomplete in a further eight patients.

Overall mortality in 206 remaining patients was: $25(12 \cdot 1 \%)$ before arrival at hospital; $45(21.8 \%)$ within two days; 85 $(41.3 \%)$ within seven days; and 146 (70.9\%) within six months. Mortality in males was $69 \%$, in females $75 \%$ and strongly increased with age.

Persisting vigilance disturbance at day 7 was followed by $98 \%$ mortality within six months. Persisting mental disturbance at day 7 resulted in $26 \%$ mortality, $44 \%$ persisting mental disturbance, and $30 \%$ complete recovery at six months.

All mentally normal patients at day 7 survived at six months. Patients with a Glasgow Coma Scale score of $>8$ at day 1 exhibited a $40 \%$ mortality at six months, which did not differ from the $\mathbf{4 2 . 8 \%}$ mortality in patients with a score of $<8$ at day 1 .

Focal neurological deficits were found in only eight patients $(3 \cdot 8 \%)$.

MYELINATED A $\beta$-AFFERENTS SIGNAL

ALLODYNIA ASSOCIATED WITH POSTHERPETIC NEURALGIA

R Baron. University of Kiel, Kiel, Germany

Postherpetic neuralgia (PHN) is often accompanied by pain to light touching (allodynia). Do myelinated $\mathrm{A} \beta$-fibres of low-threshold mechanoreceptors or sensitised unmyelinated nociceptive C-fibres signal this allodynia?

Stimulation and sensitisation of polymodal nociceptive $\mathrm{C}$-fibres cause pain and due to afferent axon reflex (AR) activation a local skin vasodilatation. To determine the involvement of C-fibres, skin blood flow and temperature within the affected segment were compared with the contralateral area. The local AR-vasodilatation to mechanical and chemical C-fibre stimulation was compared with the reaction to allodynic stimulus of $20 \mathrm{~s}$. A patient with allodynia associated with PHN (right $\mathbf{T H}$ 6.7) was studied using a Laser-Doppler perfusion monitor (Periflux Pf3, standard probe, $12 \mathrm{kHz}$, time constant $1.5 \mathrm{~s}$, technical zero subtracted).

In the allodynic area resting blood flow was $50 \%$ less and skin temperature $0: 6^{\circ} \mathrm{C}$ lower than on the contralateral side.

Mechanical and chemical (local application of histamine) C-fibre stimulation increased skin perfusion considerably due to AR-vasodilatation. The allodynic stimulus did not cause any blood flow change.

These two lines of evidence indicate that sensitised nociceptive C-fibres are not involved in this patient's allodynia. Probably low-threshold mechanoreceptors with $A \beta$-fibres signal the allodynia. Central changes in the dorsal horn may occur after zoster infection that strengthen the synaptic ties between central pain signalling pathways and low-threshold mechanoreceptive afferents.
THE CLINICAL, METABOLIC, AND HISTOPATHOLOGICAL CORRELATES OF OROFACIAL DYSPRAXIA

AM Kennedy, PJ Tyrrell, L Kartounis, RSJ Frackowiak, L Findlay, $P$ Lantos, MN Rossor. MRC Cyclotron Unit, London, UK

Progressive loss of speech output with orofacial dyspraxia has been described due to focal degeneration of the frontal lobes. Focal cognitive deficits may herald dementia and a variety of disease processes may be responsible for this, which include Pick's disease, Alzheimer's disease and spongiform changes. We report the clinical, metabolic, and histopathological data of three patients in whom orofacial dyspraxia was the presenting feature. One patient had evidence of amyotrophy and fasciculation. Longitudinal neuropsychological assessment showed a progressive decrease in speech output as well as more generalised cognitive deficit. All patients underwent PET which demonstrated marked frontal hypometabolism (steady state ${ }^{15} \mathrm{O}$ method). Two patients had histopathological changes consistent with Pick's disease and one showed spongiform degeneration providing evidence for heterogeneity of the underlying histopathological cause of orofacial dyspraxia in dementia.

KENNEDY'S SYNDROME WITH ARTERIAL HYPERTENSION AND GOITRE

C Hartard, H-J Stürenburg, K Kunze. University of Hamburg, Hamburg, Germany

Kennedy's syndrome is an X-linked recessive multisystem disease with bulbospinal muscular atrophy, sensory neuropathy, gynaecomastia, endocrinopathy, hand tremor and a reduced glucose tolerance in many patients. We report a patient with Kennedy's syndrome with additional signs of disturbances in his thyroid and his catecholamine metabolism and with arterial hypertension.

The 65-year-old man developed a slowly progressive proximal weakness of both arms and legs, dysarthria, dysphagia, generalised fasciculations, arterial hypertension, nervousness, gynaecomastia, and a fine tremor of the hands beginning at 45 years. Motor and sensory nerve conduction velocities were slightly reduced. Muscle biopsy showed neurogenic atrophy together with secondary myopathic changes. Endocrine studies revealed normal serum values for testosterone, LH, 17- $a$-progesterone, prolactin, HGH, $\beta$-oestradiol and a markedly increased FSH level. Urinary catecholamine excretions were increased. Thyroid function test showed normal serum T3 and T4 levels but reduced basal TSH and TRH stimulated TSH levels and an enlarged thyroid gland. Glucose tolerance was reduced.

Arterial hypertension was also observed in one of Kennedy's patients and in three of Tsukagoshi's patients. Our findings might hint of disturbances also in the metabolism of thyroid hormones and catecholamines associated with the disease.

NARCOLEPSY-LIKE SLEEP EPISODES AS THE ONLY MANIFESTATION OF EPILEPSY YM Hart, MS Schwartz, MM Brown. Atkinson Morley's Hospital, London, UK

Many types of epilepsy are provoked by sleep, and in some patients their only attacks occur with sleep or in drowsy states. 
Although periods of drowsiness may be seen in patients with epilepsy, particularly following an ictal event, the overwhelming urge to sleep has not been recognised as a manifestation of ictal activity. We present the cases of two middle-aged women who presented with a history of episodes of daytime drowsiness and sleep. There was no history of loss of consciousness and no evidence for narcolepsy or sleep apnoea. Both showed temporal lobe spikes in their EEGs, and in both cases symptoms were abolished by antiepileptic therapy. We consider that these cases represent narcolepsy-like sleep episodes due to seizure activity in the temporal lobes.

SULPHATION OF CATECHOLAMINES AND PHENOLS IS REDUCED IN MIGRAINE

AL Jones, MWH Coughtrie, RC Roberts. University of Dundee, Dundee, Scotland, UK

Many of the compounds, both endogenous (e.g. catecholamines) and dietary (i.e. tyramine), associated with the pathogenesis of migraine are conjugated with sulphate. These metabolic reactions, which normally reduce the biological activity of a compound, are catalysed by the phenolsulphotransferases (PSTs), present in most body tissues, including the liver, brain and platelets.

Previous work has shown that in patients with migraine, platelet phenol PST ( $P$ PST) activity is decreased, but monoamine PST (M-PST) is normal. We have investigated PST activities in platelets from migraine patients, but have used kinetic analysis of the two enzyme activities in order to gain further insight into the role which the PSTs play in susceptibility to migraine. This approach also allows more reliable comparison between different samples.

Our preliminary data show that $\mathrm{V}_{\max }$ and $\mathrm{K}_{\mathrm{m}}$ for both P-PST and M-PST are decreased approximately $40 \%$ in migraine patients compared with non-migraine sufferers. These results suggest that while the catalytic capacity of the enzymes are reduced, there is increased affinity for the substrates. This is the first demonstration that the ability to sulphate catecholamines is impaired in migraine sufferers. In addition, we have shown that phenylethylamine, implicated in dietary migraine, is a competitive inhibitor of M-PST.

Reduced sulphation of catecholamines may therefore provide a biochemical explanation for susceptibility to migraine.

NITRTE PRODUCTION BY RAT MICROGLIAL CELLS

J Zielasek, M Tausch, KV Toyka, HP Hartung. University of Würzburg, Würzburg, Germany

Brain macrophages have been implicated in the pathogenesis of multiple sclerosis. Macrophages may damage surrounding white matter by exerting toxic effects on oligodendrocytes or by phagocytosing myelin. Macrophages secretes a large variety of possible toxic substances, and recently much has been learned about the tumour and parasite cytotoxic effect of the secreted nitrogen oxide (NO) radical, which is liberated from L-arginine by activated macrophages. We studied whether microglia/brain macrophages from neonatal Lewis rats were able to activate this biochemical pathway. We found significant secretion of the stable end product nitrite into supernatants of microglia/macrophages/ micro-well cultures ( $>90 \%$ viability, $>90 \%$ ED1-positive) after 48 hours of incubation with $100 \mathrm{U} / \mathrm{ml}$ of interferon- $\gamma$ (IFN- $\gamma$ ) (34 (16) $\mu \mathrm{M}$, mean (SD), or $10 \mu \mathrm{g} / \mathrm{ml}$ of lipopolysaccharide (32 (11) $\mu \mathrm{M})$ as compared with unstimulated cultures (4 (4) $\mu \mathrm{M})$ and to astrocyte-enriched glial cells (>90\% GFAP-positive; with IFN: 6 (5) $\mu \mathrm{M}$, with LPS: $16(6) \mu \mathrm{M})$. Both IFN- $\gamma$ and LPS-induced production of nitrite were inhibited by a specific competitive inhibitor of nitrite synthase, $\mathrm{N}$-monomethyl-arginine $(100 \mu \mathrm{M})$, and were dependent on the cell number and incubation time. These results demonstrate that activated newborn rat microglia/brain macrophages are capable of activating the NO radical-forming biosynthetic pathway. Further studies are needed to demonstrate the production of NO and/or nitrite in active multiple sclerosis lesions.

OPTIC NERVE SHEATH DECOMPRESSION

WT Green, MD Sanders. National Hospital for Neurology \& Neurosurgery, London, UK

Optic nerve sheath incision has now been in vogue for the past decade, and has proved effective in the prevention of visual loss in papilloedema. The operation has a low morbidity, is relatively simple to perform, and the patient is in hospital for less than a week. In some cases this may be the only procedure capable of preventing visual loss (eg, failed shunts, metastatic disease, dural arteriovenous malformations, and Engleman's disease).

In certain cases of progressive bilateral visual loss due to chronic ischaemic papillopathy, an improvement in vision has been described following this procedure. Our experience in 17 patients and the mechanism by which improvement occurs will be considered. Finally the value of this approach in biopsy of the optic nerve and sheath will be considered.

GAMMAGLUTAMYL TRANSFERASE ISOFORMS IN PATIENTS TAKING ANTICONVULSANT DRUGS

MJ O'Kane, M Watt, SA Hawkins, ER Trimble. Queen's University of Belfast and The Northern Ireland Neurological Service, Belfast, UK

Increased serum gammaglutamyl transferase (GGT) is a sensitive marker of hepatobiliary disease. However, interpretation may be difficult in patients taking enzyme inducing anticonvulsant drugs. GGT forms complexes with lipoproteins to give a range of different molecular weight isoforms in serum. The isoform pattern may have diagnostic value. Using molecular exclusion chromatography and polyanionic precipitation of lipoproteins we investigated the factors influencing the GGT isoform profile in 50 patients with elevated serum GGT activity induced by anticonvulsant drugs. Three major isoforms were found in each patient: form I-GGT complexed with LDL/VLDL; form II-GGT complexed with HDL; form III-free uncomplexed GGT. There was considerable between-individual variation in the proportion of total serum GGT associated with each form: form I-10\%(6-33\%) mean (range), form II-77\% (52-91\%), form III-14\% (4-33\%). There was a significant correlation between HDL cholesterol when expressed as a fraction of total cholesterol and the proportion of total serum GGT complexed with HDL ( $r=0.37$, p $<0.05)$. This suggests that the relative concentrations of lipoproteins are important in determining the GGT isoform pattern Changes in the isoform pattern in disease therefore might only reflect changes in lipoprotein distribution.

ARE IPSILATERAL MOTOR PROJECTIONS ACTIVATED DURING RECOVERY FROM STROKE?

B Boroojerdi, U Milde, A Ferbert. RWTH Aachen, Aachen, Germany

Focal magnetic stimulation of the motor cortex of one hemisphere normally results in purely contralateral responses, at least in small hand muscles. It is, however, unknown whether ipsilateral projections can be activated during recovery from weakness due to stroke.

We used an eight-shaped magnetic coil to stimulate the unaffected motor cortex 6-7 $\mathrm{cm}$ lateral of $\mathrm{Cz}$ in 10 patients 3-28 months after an ischaemic hemispheric infarction. All patients had initially hemiparesis of various degree. At the time of magnetic stimulation paresis had totally resolved in seven patients and partially in the remaining three. Recordings were made from both first dorsal interosseus muscles (FDI). In only one patient were we able to detect a small response in the FDI of the paretic side to stimulation of the non-affected hemisphere whereas stimulation of the affected hemisphere gave rise to responses in (previously) paretic FDI muscles of all patients. From these results we conclude that during recovery from weakness in small hand muscles ipsilateral projections play only a minor role. As the degree of paresis in our patients was comparably mild, we can not answer whether this also holds true for more severe paresis.

A SURVEY OF NEUROLOGICAL DISABILITY AT A DISTRICT GENERAL HOSPITAL ED Playford, $\mathbf{P}$ Crawford, PS Monro. Atkinson Morley's Hospital, London, UK

Neurological disease accounts for $20 \%$ of all admissions to medical wards, but the number of inpatients with neurological disability is unknown. We performed two surveys at a Teaching District Hospital to identify all adult inpatients with a neurological disorder. For these patients the age, diagnosis, length of stay, disability and the reasons for admission and for remaining in hospital were recorded.

Over $40 \%$ of inpatients on medical wards and $4 \%$ on surgical wards had a neurological disorder. Stroke was the commonest neurological diagnosis accounting for $36 \%$ of neurological disorders and $22 \%$ of all patients on medical wards. Other neurological diagnoses included dementias $10 \%$, degenerative disorders $10 \%$, epilepsy $7 \%$, demyelination $4 \%$, and head injuries $3 \%$. Neurological patients had a mean stay of 80 days. A total of $21 \%$ of inpatients were waiting for appropriate accommodation. Seven patients under age 65 stayed more 
than six months awaiting long-term care.

Neurological disorders contribute significantly to the inpatient workload. Identifying those requiring rehabilitation, and their concentration on one ward could improve the quality and efficiency of their care and ease teaching. Approximately 20 beds would be required. Neurologists cannot see every patient with a neurological disorder and so general physicians should be adequately trained in neurology.

SINGLE GENE NEUROLOGICAL DISORDERS IN SOUTH WALES

JC MacMillan, PS Harper. Institute of Medical Genetics for Wales, Cardiff, UK

Inherited neurological disorders have been the focus of extensive investigation in recent years through the use of recombinant DNA technology. The technique of positional cloning ("reverse genetics") has provided a means of identifying the genetic basis of several debilitating familial neurological diseases and engendered the hope that some of these disorders may eventually be "treatable". In those disorders where the gene product and its function is not yet known accurate chromosomal localisation of the relevant gene (or genes) increases the reproductive options for couples at risk by permitting prenatal diagnosis through linkage analysis and termination of affected foetuses. Direct mutation screening is already available for Duchenne and Becker muscular dystrophies and type 1 neurofibromatosis. Presymptomatic and prenatal assignment of affection status is available for myotonic, facioscapulohumeral and Emery-Dreifuss muscular dystrophies, and for Huntington's disease. These services are currently available in regional molecular genetic units in the UK. Future applications are likely to see prenatal testing for one type of hereditary motor and sensory neuropathy and the proximal spinal muscular atrophies of childhood. In any attempt to assess the likely impact of DNA diagnostic services in this field it is essential to know the frequency of these diseases in the population. A hospital based epidemiological study of the major single gene neurological disorders in South East Wales has estimated the minimum overall prevalence of these disorders to be 58.6 per 100000 population. These individuals are now in a position to benefit from these recent molecular genetic advances.

THE INTERNATIONAL STROKE TRIAL TO TEST ASPIRIN AND HEPARIN IN ACUTE ISCHAEMIC STROKE

RI Lindley on behalf of the International Stroke Trial Collaborative Group. University of Edinburgh, Edinburgh, Scotland, UK

The International Stroke Trial is a large multicentre randomised trial which aims to recruit over 10000 patients with acute ischaemic stroke (with randomisation within 48 hours of onset of symptoms) in 200-400 centres worldwide. Oral aspirin (or suppository) and two doses of subcutaneous heparin will be tested in a $3 \times 2$ factorial design. To obtain such large numbers it is necessary to have an extremely simple and robust design. To achieve this a large scale pilot study in four United Kingdom and six European centres started on 14th January 1991. By 1st June 199148 patients had been randomised. A total of $86 \%$ of acute stroke patients were randomised with- in 24 hours of onset of symptoms, $40 \%$ within 12 hours. In the Edinburgh centre $47 \%$ of acute stroke patients admitted to hospital have been randomised into the trial, the main reasons for exclusion being contraindications to aspirin or heparin, known intracerebral haemorrhage, or greater than 48 hours since the onset of symptoms.

These data confirm that large simple trials early in acute ischaemic stroke are practicable. The pilot study will continue until late 1992 when there should be sufficient safety data to commence the main study.

CRANIAL COMPUTERISED TOMOGRAPHIC PATTERNS IN DIFFERENT STROKE AETIOLOGIES

H Mast, F Nüssel, H Völler, K Schröder, T Heinsius, $P$ Marx. Free University of Berlin, Berlin and University of Erlangen, Erlangen, Germany

Cranial CT patterns have been claimed to be an indicator for different stroke aetiologies. We tested this hypothesis in a prospective study in 118 consecutive patients in whom cranial CT, echocardiography, Doppler sonography, and electrocardiography were performed. 39 (33\%) patients had ECG and/or ECHO findings indicative of cardioembolic stroke. An ipsilateral carotid artery stenosis was found in $18(15 \%)$ patients. $58(49 \%)$ had a history of hypertension. Thirty-six (31\%) patients showed none of these aetiological factors (N-group). A large group ( $n=30,25 \%$ ) had multiple aetiological factors. In 52 (44\%) patients only one aetiological factor was evident.

Pial artery infarctions and small deep infarctions (lacunae) were equally frequent in hypertensive patients, the group with a potential cardioembolic source, and the patients with multiple aetiological factors. Hypertensive patients did not show an excess of small deep infarctions. Likewise, the group of presumed cardioembolic stroke did not exhibit more pial artery infarctions.

In conclusion, a single pial artery infarction is not indicative of cardioembolic stroke. Multiple pial artery infarctions, however, might correlate with a cardioembolic source.

The finding of different cranial CT patterns in patients with a possible cardiac source of embolism either reflects arteriosclerotic disease affecting both heart and brain vessels or a greater morphological variety of stroke pattern in cardiac embolism than previously assumed.

Small deep infarctions (single or multiple) are not indicative of "hypertension microangiopathy".

HAEMORHEOLOGICAI DEFICITS ARE A SECONDARY RISK FACTOR FOR STROKE E Ernst, K Resch. University of Vienna, Vienna, Austria

The hypothesis that haemorheological abnormalities might represent a bad prognostic sign in stroke was tested in a prospective trial on survivors of a first stroke. A total of 625 patients were examined 7.4 months after the acute event. Native and haematocrit-standardised blood viscosity at three shear rates, its main determinants, haematocrit, plasma viscosity, fibrinogen as well as erythrocyte sedimentation rate (ESR), total leukocyte count, total cholesterol, triglycerides, blood pressure, and body mass index (BMI) were measured. Patients were followed up for two years. By then 85 patients had suffered a second stroke, myocardial infarction or had died due to a cardiovascular event. Matched pairs were formed between patients with and without such re-events. (Matching criteria: total cholesterol, triglycerides, fasting blood sugar, BMI, sex, age, blood pressure, smoking habits, time interval between first stroke and the blood tests as well as concomitant diseases). Such matching was possible in 60 cases. Only native blood viscosity, plasma viscosity and fibrinogen were significantly higher in patients who had suffered a re-event compared with those who had not. It is concluded that in survivors of a first stroke, hyperviscosity of blood and plasma as well as hyperfibrinogenemia are risk factors for further cardiovascular events.

\section{ENDOTHELIAL CELLS IN VASCULITIS OF} PERIPHERAL NERVE AND SKELETAL MUSCLE PK Panegyres, RJ Faull, GR Russ, PC Blumbergs. Queen Elizabeth Hospital, Adelaide, Australia

In our previous studies of the pathogenesis of vasculitis in nerve and muscle we classified patients into three groups: I-multisystem vasculitis; II-multisystem disease with vasculitis detectable only in muscle and nerve; and III-vasculitis localised to nerve and muscle. Ten of 23 patients had vasculitis in both nerve and muscle biopsies Immunotypic and immunofluorescence analysis of the vasculitic lesions indicated that the pathogenesis is heterogeneous and involves cell-mediated and immune complex mechanisms in all three groups. We investigated the role of endothelial cells in the pathogenesis of vasculitis by exploring the expression of endothelial adhesion molecules using monoclonal antibodies and immunoperoxidase methods. We studied endothelial cells in frozen sections from nerve and muscle biopsies obtained from seven patients from the three groups. The expression of adhesion molecules was related to major histocompatibility antigens (MHC) and to the presence of antiendothelial cell antibodies (AECA) in blood using an ELISA. We have demonstrated increased expression of the intercellular adhesion molecule ICAM-1 in all patients compared with controls. This correlated with increased MHC class I and II antigen expression. AECA were found in low titre in two of 12 patients. These findings suggest that endothelial cells, through upregulation of their adhesion molecules and MHC antigens, play a fundamental role in mediating tissue injury. This mechanism probably represents the final common pathway of vasculitic activation in all patient groups and may be provoked by cell-mediated and immune complex factors. Our data does not support a role for AECA in this process.

EARLY GENERALISATION OF DENERVATION IN AMYOTROPHIC LATERAL SCLEROSIS

K Kunze, G Winkler, G Thayssen. University of Hamburg, Hamburg, Germany

In 120 patients with proven amyotrophic lateral sclerosis (48 women, 72 men; $34-82$ years) a primary bulbar manifestation in $32.5 \%$, a primary arm manifestation in $22 \%$, and a primary leg manifestation in $18 \%$ was found besides primary generalisation. Clinical examinations were performed 
after 2-84 months (mean 17). The most impressive feature was a variable but fast generalisation. With regard to this, muscle biopsies of the tibialis anterior muscle already showed a neurogenic affection partially of reticular type in a group of patients with primary bulbar or arm/hand localisation, without signs in the legs, who had been seen early in their clinical course. This confirmed earlier examinations of a group of 100 patients (50 women, 50 men) with different clinical sign combinations and different progression of clinical course. Common to all patients in different groups was a more or less fast generalisation. Spastic types seemed to have a slower progression. So by muscle biopsy a very early generalisation could be detected even in those cases in which no clinical or electromyographical signs could be proven.

\section{A STUDY WITH THE GANGLIOSIDE GM1} AGAINST AMYOTROPHIC LATERAL SCLEROSIS D Ritter, WF Haupt, WD Heiß. University of Köln, Köln, Germany

For patients with amyotrophic lateral sclerosis a prospective phase II study was performed giving $100 \mathrm{mg}$ intravenous GM1 (FIDIA) daily for 20 days and re-examining them six months later.

A total of 61 consecutive patients (34 men, 27 women) were diagnosed with amyotrophic lateral sclerosis by a programmed evaluation (including MRC strength testing of 30 muscles, Norris disability score, EMG, muscle biopsy and others). Seventeen had bulbar onset. Mean duration of disease at treatment was 10.8 months. Forty-five were treated after informed consent. One patient showed mild allergic reaction and treatment was stopped. Twenty patients were studied after excluding patients with severe concurrent diseases (six), atypical findings (four), death within six months (eight) or missing data (seven).

The disability score at treatment was 96 of 120 , the muscle strength 134 of 150 possible numbers. The disability score after six months decreased $15 \%$, the muscle strength $12 \%$. Mean survival time was $22 \cdot 2$ months. We found no significant difference for any investigated parameter between the treated and the untreated group. Patients with concomitant disease and atypical findings showed similar clinical courses. The results were comparable to other reports. Limited by the power, no beneficial effect of this therapy was observed, nor any unwanted major side effects. As a positive side effect evaluation for the detection of "symptomatic" amyotrophic lateral sclerosis and follow-up care improved.

A PILOT STUDY OF SMELL IDENTIFICATION IN MOTOR NEURON DISEASE

$M$ Elian. Oldchurch and Charing Cross Hospitals, London, UK

Sensory impairment was recently identified in Parkinson's disease, Alzheimer's dementia, and motor neuron disease (MND) previously thought to involve higher mental functions and the motor pathways. An impairment of the ability to smell was found in the sporadic form of Alzheimer's and Parkinson's disease and in patients with the Parkinson dementia complex in Guam and in some of their healthy relatives. The ability to smell was not investigated in MND.
Olfactory research is now greatly facilitated by a repeatable and well standardised method: the University of Pennsylvania Smell Identification Test (UPSIT). The purpose of this pilot study was to compare the ability to identify smells in MND sufferers and controls, well matched for age, sex, and smoking habits. The considerable difference found between patients' and controls' ability to identify smells was statistically significant at the $\mathbf{0 . 0 0 5}$ level. Is the olfactory impairment a result of MND or is it related to its cause, or is the relationship more complex? The answer is not yet clear and more research is planned to explore this new avenue. The findings indicate the presence of a vulnerable olfactory system in MND, which may be due to a genetic predisposition.

MOTOR NEURON DISEASE ON THE INCREASE: REALITY OR FALLACY?

$M$ Elian, G Dean. Oldchurch and Charing Cross Hospitals, London, UK and The Research Board, Dublin, Eire

An increase in the number of people suffering from motor neuron disease (MND) was reported in recent years from various countries in Europe, Asia, and North America, having occurred over the last three decades. This was surprising as MND had a reputation of being evenly distributed among peoples of the world and to remain constan throughout the decades. For a variety of reasons we are confident that mortality from $M N D$ is a reliable measure for mor bidity. We have therefore studied the reported mortality from MND in different populations, and the changes that occurred over the last two decades in England and Wales, the Republic of Ireland, Australia, and New Zealand, using multiple sclerosis for comparison. Our results confirm a considerable increase; most but not all the increase was reported in the middle aged and elderly. Is this increase genuine or the result of better ascertainment and improved care? Have environmental factors characteristic of our century become more widespread and/or active causing more people to contract and die from MND? Has the doctor's ability or habit to diagnose MND changed? Answering these questions may eventually lead to the cause of MND; until then it will help us to plan for the health care of the coming decades.

AMYOTROPHIC LATERAL SCLEROSIS AND DEMENTIA OF ALZHEIMER TYPE: A CASE REPORT

P Vieregge, E Reusche, $M$ Müller, K Ogomori. University of Lübeck, Lübeck and Ludwig-Maximilian University, Munich, Germany

A woman with concurrent sporadic amyotrophic lateral sclerosis (ALS), mild diabetic neuropathy, and dementia died aged 59 after three years. Dementia had occurred at the time of onset of motor disturbances and presented as frontal lobe dementia (FLD) with personality changes, memory deterioration, and slowed mental processing predominantly. Pathology confirmed typical motor neuron disease of ALS and revealed marked atrophy of the frontal lobe with multiple senile plaques in the frontal, temporal, parietal, cingulate and insular cortex as well as in the hippocampus. Here, in addition, the diffuse spread of neurofibrillary tangles confirmed by silver staining and immunohistochemistry was judged as Alzheimer's disease (DAT). While FLD with non-specific microscopical features may occur, DAT is very rarely seen in familial or sporadic ALS. It raises the question whether this association may have a common pathogenesis or has appeared coincidently.

GENE MAPPING AND MUTATION DETECTION IN HEREDITARY MOTOR AND SENSORY NEUROPATHY TYPE I

S Malcolm, PJ Hallam, J Berciano, AE Harding. Institute of Child Health and Institute of Neurology, London, UK and Hospital Nacional Marques de Valdecilla, Santander, Spain

The demyelinating type of autosomal dominant hereditary motor and sensory neuropathy (HMSN type I) has been shown to be genetically heterogeneous, with a major gene mapping to chromosome 17

A set of probes, D17S122, D17S125, and D17S124 (the 400 series) show tight linkage to this HMSN I locus. Van Broeckhoven and Raeymaker recently showed that the locus D17S122 is duplicated in Belgian and Dutch HMSN families, and we have confirmed this in $7 / 7$ British and Spanish kindreds. No normal individuals have been found with this duplication. The duplication can readily be seen using the Mspl polymorphism of the probe p409R3. However, recombinants still exist between the polymorphism of 409R3 and the duplicated band, suggesting that the duplicated region is of considerable size.

These observations will have considerable impact in the diagnosis of hereditary motor and sensory neuropathies, in both symptomatic and at risk individuals, and have potential application in prenatal diagnosis.

MYOTONIC PUPILS AND HEREDITARY MOTOR AND SENSORY NEUROPATHY

DJ Dick, TJ Walls, RDC Elwes, $P$ Hudgson. Newcastle General Hospital, Newcastle upon Tyne, UK

Five patients from three generations were found to have hereditary motor and sensory neuropathy (HMSN) type II and myotonic pupils. Each member of the family was examined clinically and neurophysiologically. One affected individual from each generation had pharmacological tests of pupillary function performed. The index case also had a sural nerve biopsy and detailed autonomic testing.

Nerve conduction studies were compatible with HMSN type II. The nerve biopsy showed features of chronic demyelination and axonal degeneration/regeneration. Autonomic testing was normal. Pupils showed no reaction to light or accommodation but constricted in response to dilute methacholine. Slit lamp examination revealed minimal irregular pupillary constriction and sectored iris atrophy. These are characteristic features of the HolmesAdie pupil.

The commonest pupillary abnormality to have been associated with HMSN is "Argyll Robertson" or miotic pupils. Myotonic pupils tend to regain their reaction to accommodation and become smaller with 
age so previous reports may have been describing end stage myotonic pupils. The Holmes-Adie pupil is often associated with depressed or absent reflexes and it is of interest that it may also be seen with HMSN.

PERIPHERAL NEUROPATHY ASSOCIATED WITH HTLV-1 INFECTION

JM Vallat, H Ramiandrisoa, M Dumas, EK Grunitsky, F Akani, F Denis. University of Limoges, Limoges, France

A total of 865 patients in hospital in four towns in West Africa (Dakar, Abidjan, Lome, Ouagadougou) were examined prospectively over a 30 month period. They had a variety of conditions, and 34 cases were HTLV-1 seropositive, i.e. a prevalence of $3.93 \%$ (general population $3-5 \%$ ). Five of these patients came from 153 cases of peripheral neuropathy (PN) (prevalence $3.26 \%$ ). Nerve biopsies were taken from two of these five patients. In both cases we observed discrete demyelinating lesions, and in one case there were light microscopical signs of inflammation. Two cases of polyradiculoneuritis were HIV-1 positive in both serum and CSF. The so called tropical spastic paraplegias included the largest number of seropositive patients with an overall prevalence of $15.8 \%$ ( 82 cases) Nerve biopsies were taken from seven of these patients, which in one case disclosed inflammatory lesions, although with no involvement of nerve fibres. This patient was HIV-1 positive in both serum and CSF The six other biopsies were normal. One patient was HIV-1 seropositive (CSF-negative) and the other HIV-2 positive in both serum and CSF. These findings together with other reports in the medical literature do not yet constitute sufficient evidence that the HTLV-1 virus alone is responsible for PN.

SIDE EFFECTS OF CHRONIC SUBCUTANEOUS ADMINISTRATION OF LISURIDE IN THE TREATMENT OF PARKINSONIAN PATIENTS WITH SEVERE MOTOR FLUCTUATIONS A Heinz, I Suchy, M Langkafel, D Pöhlau, L Schöls, N Przuntek. University of Bochum, Bochum and Schering AG, Berlin/Bergkamen, Germany

Twenty-nine patients with severe motor fluctuations were treated with subcutaneous lisuride infusion $(0.99$, range $0.3-2.0 \mathrm{mg} /$ 48 hour). Mean daily dose of levodopa at baseline was 512 (SD 273) $\mathrm{mg}$ showing no significant reduction during the whole observation period. Initial results, which consisted of mean reduction of "off" phases by 3-4 hours per day and improved walking ability in "off" and in "on" remained unchanged throughout the observation period. The observation period was three months for 25 patients, six months for 17 , 12 months for seven and 36 months for three. One patient with a history of heart failure had to interrupt treatment because of peripheral oedema. Another patient stopped lisuride because of psychosis. Reduction of lisuride in 10 patients-and additional promethacine in three-abolished psychic disturbances and patients thereafter continued lisuride infusions. Seven patients interrupted treatment for various reasons (unsatisfactory response in three patients, difficulty in handling the pump in three, and cosmetic reasons in two). Nausea and vomiting in two patients were treated with domperidone within the first three months after lisuride infusions were initiated. Orthostatic dysregulation, retrosternal pain and tachycardia in one patient each were treated symptomatically.

CONSTIPATION IN PARKINSON'S DISEASE WH Jost, $\mathrm{K}$ Schimrigk. University of Saarland, Homburg, Germany

Constipation is known as a frequent symptom in Parkinson's disease. A quantification of this functional disturbance has not yet been made. In our study colonic-transit was measured in 20 cases. The patients received 10 inert radio-opaque pellets over six days. Abdominal radiography was carried out on the seventh day. Transit time was determined. During the examination medication was not changed. The patients received low-fibre diet and no laxatives. In 16 cases there was a delayed average colonic passage of 5-7 days. Four patients had a transit time within normal limits. The delayed transport affected all sections of the colon. A dependance on age, sex, presentation of the disease, physical activity, or medication could not be seen. In our opinion lesions in the autonomic nervous system are responsible for this disturbance.

RIGIDITY, AKINESIA, AND DYSPHAGIA IN OPCA AND THE EFFECT OF CONTINUOUS LISURIDE INFUSION

A Heinz, J Wöhrle, W Kuhn, P Klotz, H Przuntek. University of Bochum, Bochum, Germany

Four patients with sporadic olivopontocerebellar atrophy (OPCA) and marked rigidity and akinesia were treated with continuous subcutaneous lisuride infusions. Mean age of the patients was 61 years, mean duration of disease 7.8 years. Mean levodopa dosage at baseline before subcutaneous lisuride was $525 \mathrm{mg}$, and all patients received additional selegiline. Two patients received oral bromocriptine $(22.5$ or $20 \mathrm{mg} /$ day) and one lisuride $(1.0 \mathrm{mg} /$ day $)$ at baseline, which was stopped when lisuride infusions were started.

The motor performance of the four patients was assessed clinically and rated using the King's College Hospital Parkinson's Disease-Rating Scale (KCHRS). Data are available before starting the lisuride infusions, at the time of discharge and after six months of treatment.

Three patients showed an overall improvement of motor performance, while in one patient only dysarthria and dysphagia improved. KCH-RS total score dropped significantly from $\mathbf{8 5 . 2 5}$ to $\mathbf{7 5 \cdot 5 0}$ (Wilcoxon test, $\mathrm{p}<0.05$ ) while treated in hospital. This result remained stable after six months of lisuride infusion. One patient stopped lisuride infusion because of difficulties in handling the pump after six months of treatment.

When the lisuride dosage was increased to improve motor performance, in three out of four patients the dysphagia and dysarthria worsened significantly, so that a dose reduction to about $50 \%$ became necessary.
ABNORMAL COLOUR PERCEPTION IN TREATED AND UNTREATED PARKINSONIAN PATIENTS W Kuhn, A Heinz, R Steinberg, T Büttner, M Langkafel, L Voss, D Bulgaru, H Przuntek. University of Bochum, Bochum, Germany

Under treatment with intravenous lisuride a patient with malignant neuroleptic syndrome reported a red line instead of a red point stimulus. This misperception was normalised by reducing the doses of lisuride. To investigate this phenomenon we developed a computer-aided assessment of colour perception. Differently coloured horizontal bars were divided into 60 vertical lines and presented on a monitor, flickering at $8 \mathrm{~Hz}$. The monochromatic lines enlarged irregularly and fusion to the original bars was complete after $10 \mathrm{~s}$. The point of fusion had to be announced by the patient.

We investigated 80 patients with Parkinson's disease and 59 controls. By measuring different colour items, a significantly faster fusion time was observed for dark green in treated and untreated parkinsonian patients in comparison with controls.

IBZM-SPECT PREDICTS RESPONSE TO APOMORPHINE IN PATIENTS WITH "DE-NOVO" PARKINSONIAN SYNDROME J Schwarz, K Tatsch, K Arnold, Th Gasser, CM Kirsch, WH Oertel. LudwigMaximillian University, Munich, Germany

A normal or increased density of striatal dopamine receptors and a positive response to dopamimetic drugs support the diagnosis of idiopathic parkinsonian syndrome (IPS). Single photon emission CT (SPECT) using the ligand ${ }^{123}$ Iodobenzamide (IBZM) allows imaging of striatal dopamine D2 receptors with high affinity and specificity. Subcutaneous apomorphine $(2-5 \mathrm{mg})$ has been shown to be a test for dopamimetic responsiveness.

A total of 31 patients presenting with early parkinsonian syndrome (Hoehn and Yahr, stage I or II) were studied. All patients had not received anti-Parkinsonian drugs before this study and had symptoms compatible with idiopathic Parkinson's disease. Nineteen patients displayed normal binding of IBZM and a positive response to apomorphine. Four patients showed normal IBZM binding and an equivocal response to apomorphine. Seven patients had decreased IBZM binding and a negative response to apomorphine. Two patients had normal IBZM binding and a negative response to apomorphine. According to this study, normal or decreased IBZM binding predicted a positive or negative response to apomorphine in 24 of $27(89 \%)$ of patients with "de novo" parkinsonian syndrome. Imaging of dopamine D2 receptors may be a useful screening procedure in "de novo" parkinsonian patients.

ACUTE POLYNEUROPATHY FROM LIGHTNING INJURY. REPORT OF A CASE WITH COMPLETE RECOVERY

CH Hawkes, J Thorpe. Ipswich Neurological Centre, Ipswich, UK

In Britain about 12 people each year are struck by lightning, of whom three or four will be killed. In survivors, although weakness lasting approximately 24 hours is well recognised, more permanent sequelae are 
infrequent and poorly understood from the pathological and clinical aspects.

We describe a case of a 19-year-old lefthanded docker who was admitted to hospital after being struck by lightning while astride a stationary motorcycle. On recovery from deep coma he was found to be quadriplegic. Subsequent developments suggested that his weakness was due to extensive peripheral nerve damage. In addition he displayed many well recognised medical complications of lightning injury including acute renal failure, rhabdomyolysis, respiratory distress syndrome, autonomic dysfunction, perforated ear drum, uveitis, and cataract. Despite all this he went on to make a complete recovery.

The neurological literature regarding lightning stroke was reviewed briefly.

DOES A RANDOM CREATINE KINASE LEVEL CORRELATE WITH PROGNOSIS IN MOTOR NEURON DISEASE?

$\mathrm{CH}$ Hawkes, J Thorpe. Ipswich Neurological Centre, Ipswich, UK

An elevated creatine kinase (CK) level in motor neuron disease (MND) is a well recognised phenomenon that occurs in about $40 \%$ cases. It is often stated that such enzyme change does not correlate with prognosis.

To elucidate this further we screened the case notes of all patients with a diagnosis of MND who had attended the Neurological Centre, Ipswich over a period of 10 years. The mean for 51 MND patients was 240 IU; range 30-1400. (Normal values: males $<120$ IU; females < 105 IU). Because the data had a log normal distribution, $\log$ CK was used in all analyses. Measurements were from time of CK measurement to time of death or censoring.

The bulbar group had a lower log CK than the non-bulbar group (geometric mean $95 \mathrm{IU} v 214 \mathrm{IU}, \mathrm{p}=0.001$, two sample $\mathrm{t}$ test). For survival analysis, parametric survival models were fitted to allow for patients who may not have died at the time of analysis. There was no influence of age on survival $(p=0.48)$ but the $C K$ level (whether in the normal range or above) did correlate with survival $(p=0.034)$. For the two variables-disease type and log CK, KaplanMeier survival curves were produced and the Log Rank test used to determine whether overall survival differed between them. Patients in the bulbar group survived the shorter period $(p=0.011)$. When abnormal $v$ normal CKs were compared, those with abnormal values showed a trend towards longer survival, just outside conventional significance levels $(p=0.07)$.

These findings suggest that in general, MND patients with raised $C K$ survive longer.

SIMILAR EEG FEATURES IN DEMENTED PATIENTS AND IN ANTICHOLINERGICALLY TREATED NORMALS SUGGEST A DYSFUNCTION OF CHOLINERGIC EEG MECHANISMS IN DEMENTIA

KP Westphal, B Grözinger, V Diekmann, C Haag, W Scherb. University of Ulm, Ulm, Germany

Loss of cholinergic activation in dementia together with more slow activity and lower alpha power as well as a reduced reactivity of the EEG during activation tasks are reported in demented patients. It was hypothesised that the anticholinergic biperidene should produce similar patterns in normal volunteers. In 20 normal volunteers biperidene ( $5 \mathrm{mg}$, intravenously) induced significantly (Wilcoxon-Wilcox test) higher delta power at frontal and central electrode positions. Theta power did not increase significantly but the theta centre frequency became significantly lower with biperidene. Alpha power decreased significantly, mainly over parietal electrodes.

Analysing the blocking effects in the EEG during voluntary movements, the functional significance with blocking of alpha and beta activity obtained over activated motor areas contralateral to the finger movement disappeared with biperidene, while theta power increased at these areas during the preparation of the movement and delta power increased at these areas during movement execution with biperidene. Apart from these results of the frequency analysis, the Bereitschaftspotential was higher with the anticholinergic biperidene. These anticholinergically induced results in normals suggest that indeed the similar features reported in demented patients might be due to changed cholinergic EEG mechanisms in dementia.

IMPAIRMENT OF INHIBITORY TEMPORALIS MUSCLE REFLEX IN WHIPLASH INJURY

M Keidel, $\mathrm{P}$ Rieschke, HC Diener. University of Essen, Essen, Germany

To verify the hypothesis of a brainstem dysfunction following imposed acceleration forces in whiplash injury we investigated the inhibitory, brainstem mediated $\mathrm{m}$. temporalis reflex (ITR) responsible for an early and late exteroreceptive supression (ES1 and ES2) of muscle activity. Fifty patients (29 women; 21 men; mean age 27.6 years, SD 8.5 ) with a cervicocephalic syndrome (headache, neck and muscle pain and/or stiffness) were included within 14 days after the whiplash injury (mean 4.7 days). Trigeminal nerve $2+3$ divisions were randomly stimulated at the right comissura labialis. Surface EMG of the ipsilateral temporalis muscle was recorded during maximal contraction. The data were compared to normal subjects $(\mathrm{n}=35$ : mean age $29 \cdot 2$ years, SD 7.7). The late EMG supression (ES2) was shortened in duration $(35.4 \mathrm{~ms}$, SD 9.2; normals: $47.6 \mathrm{~ms}$, SD 6.6 ; p < $0.0001)$ with a delayed onset $(52.4 \mathrm{~ms}, \mathrm{SD}$ $7 \cdot 4$; normals: $47.9 \mathrm{~ms}$, SD $5 \cdot 2$; $<<0.006$ ) and an early ending $(87.8 \mathrm{~ms}$, SD 9.9 ; normals: $95 \cdot 6$, SD 8.3 ; p $<0.0002$; Wilcoxon test). The index of ES2:ES1 was reduced (1.85 SD 0.5; normals: 2.9 , SD 0.5 ; p < $0.0001)$. The onset of ES1 $(12.2 \mathrm{~ms}, \mathrm{SD}$ $2 \cdot 1$; normals: $12.6 \mathrm{~ms}, \mathrm{SD} 2 \cdot 2$ ) and duration of the following EMG segment preceding ES2 $(20.7 \mathrm{~ms}$, SD 6.9; normals: 18.2 , SD 5.3) did not differ significantly. We conclude that there is a direct or indirect functional alteration of the neuronal structures involved in the reflex loop due to acceleration forces in whiplash injury. Despite the discussion of a malfunction of pontobulbar interneurons or proprioceptive input, the pathophysiological mechanism underlying the altered exteroceptive suppression is not fully elucidated. The pathogenesis of post-traumatic headache following whiplash injury and headache of the muscle tension type seems to be closely related, as in the latter comparable results have been reported.

ANTICHOLINERGIC EFFECT ON EVENTRELATED POTENTIALS: A PILOT STUDY WITH BIPERIDEN

G Heinz, A Haaß, M Karst. University of Saarland, Homburg, Germany

The event-related potentials (ERP) were elicited by a competitive stimulation with checkerboard reversal patterns: the target stimuli (counting) were the pattern reversals in the lower half of a monitor (random ISI, 4-10 s); the non-target stimuli (ignore) were the reversals in the upper half (constant ISI, $3.6 \mathrm{~s})$. The ERP (1500 ms) were recorded from $\mathrm{Fz}, \mathrm{Cz}$ and $\mathrm{Pz}$ (BP filter $0.02-100 \mathrm{~Hz}$ ). After a drug-free examination, the probands (nine men, two women, age 23-48 years) received $5 \mathrm{mg}$ biperiden intravenously. The ERP were re-examined 10 minutes later.

All probands showed a decrease in P300 amplitude. The P300 latencies, and all components before $300 \mathrm{~ms}$ and later than $550 \mathrm{~ms}$ of the ERP remained unaffected. This effect was pronounced in $\mathrm{Pz}$ recordings. The ERP subsequent to non-target stimuli remained unchanged. In paralle with the decrease in P300 waves, we found a significant increase in counting mistakes.

Though a specific effect on the generators of the $\mathrm{P} 300$ wave by anticholinergic agents could be suspected, a more generally impaired CNS performance under the acute adminstration of biperiden has to be assumed.

FUNCTIONAL EFFECTS OF NERVE INJURY AND AMPUTATION

S Braune, W Schady. Manchester Royal Infirmary, Manchester, UK

Dynamic changes in somatosensory cortical maps occur in animals subjected to peripheral nerve transection, with expansion of the cortical representation of areas adjacent to the numb patch of skin. It is not known whether similar changes occur in humans, and if so, whether increased cortical representation improves tactile acuity in the skin surrounding the anaesthetic zone. We have addressed this issue by (a) recording brain maps of somatosensory evoked potentials (SEP) to digital stimulation, and (b) performing multimodal quantitative sensory tests in 12 patients with traumatic division and subsequent repair of the median or ulnar nerves, and in six patients who had had a finger amputated. There was no conclusive evidence of expansion of the SEP maps from the fingers adjacent to the numb or amputated digit. As expected, sensory thresholds to touch, vibration, and two point discrimination were raised in the territory of the injured nerve, more so in patients with recent injuries. Sensitivity was not heightened in the area adjacent to the anaesthetic zone or in the stump in the case of amputees. For certain functional tasks such as intensity coding and localisation of touch there was some evidence of CNS adaptation to loss of peripheral input. Changes in the somatosensory cortex in response to nerve injury have only slight measurable effect. 
SUCCESSFUL TREATMENT OF HEREDITARY SPASTIC PARAPLEGIA WITH INTRATHECAL BACLOFEN-CONTINUOUSLY ADMINISTERED WITH AN IMPLANTABLE PUMP

D Poehlau, G Amoiridis, A Heinz, S Ruhmann, H Przuntek. Ruhr-University, Bochum, Germany

A 56-year-old male patient had chronic progressive hereditary spastic paraplegia for about 12 years. He was not handicapped by paresis; but because of his spasticity he could walk only with a painful stiffness, with the help of a stick and for short distances.

Physiotherapy and oral treatment with baclofen $(50 \mathrm{mg} /$ day $)$ in combination with memantine $(40 \mathrm{mg} /$ day) failed to reduce spasticity. Higher doses of antispastic drugs lead to potency disturbances.

An intrathecal treatment was started. A trial dose of $50 \mathrm{~g}$ baclofen, admitted into the lumbar subarachnoid space by lumbar puncture, showed impressive reduction of spasticity. A pump for chronic intrathecal treatment with baclofen was implanted (infusaid model 400).

Now, 30 months after implantation of the pump, the patient is still able to walk quite normally for some hours, and he can ride on his bicycle. His gait is a little ataxic, but he is not really handicapped in his daily life. No complications occurred. The dose of baclofen had to be raised from an initial $75 \mathrm{~g}$ to $160 \mathrm{~g}$.

In our opinion, this kind of treatment should be tested whenever spasticity cannot be treated sufficiently by physiotherapy and oral medication.

\section{INTRATHECAL TREATMENT WITH}

TRIAMCINOLONE-ACETONIDE OF PATIENTS WITH DISSEMINATED ENCEPHALOMYELTTIS D Poehlau, J Wagener, K Koehler, G Amoiridis, H Przuntek. Ruhr-University, Bochum, Germany

A total of 43 patients with disseminated encephalomyelitis of the chronic progressive type were intrathecally treated with triamcinolone acetonide. Twice a week we injected $40 \mathrm{mg}$ of the corticoid into the lumbar subarachnoid space by lumbar puncture. In $60 \%$ of the patients we found an improvement of the motor, sensory, and co-ordination functions. Some complications arose as will be shown, but no patient worsened during this treatment.

The immunoglobulin $G$ level of the cerebrospinal fluid (CSF) was significantly reduced, the blood brain barrier remained undisturbed and the number of white cells in the CSF was reduced. The CSF level of triamcinolone acetonide was 50-1000 times higher than the plasma level.

In our opinion this treatment is an acceptable and effective method for treating this subgroup of patients with poor prognosis.

THE SCOTTISH MOTOR NEURONE DISEASE REGISTER: A PROSPECTIVE STUDY OF MOTOR NEURONE DISEASE IN SCOTLAND:

METHODOLOGY, DEMOGRAPHY AND CLINICAL FEATURES OF INCIDENT CASES FOR 1989 AM Chancellor, RJ Swinger, CP Warlow for the Scottish Motor Neurone Disease Study Group. University of Edinburgh, Edinburgh, UK

The lessons from the Western Pacific form of motor neuron disease (MND), the low concordance rate in twin studies and chang- ing trends in mortality suggest that aetiological clues for MND might be derived from epidemiological studies. The Scottish Motor Neurone Disease Register (SMNDR) is a prospective, collaborative, population based study of MND in Scotland. It is the first study of MND of its kind and is based on a disease register recruiting patients from multiple sources with the aim of achieving complete case ascertainment in Scotland. Detailed clinical information is collected on each patient and follow up is complete. In this paper we report the methodology, incidence data and simple clinical features of patients newly diagnosed in the first year. A total of 114 patients $(62 \mathrm{men})$ were diagnosed in 1989. The crude incidence was $2 \cdot 24 / 100000$ per year, age-specific incidence rates rose sharply into the 65-74 year age group and the high rate was maintained into the 75-84 year age group. The mean age at onset for women (68.0 years) was significantly older than for men (62.5 years). The relative risk of bulbar involvement at onset for females when compared to males was $3.05(95 \% \mathrm{CI}$ 1.6 to 6.0 ). A non-significant, non-random distribution was observed between geographical regions but more reliable information in this regard will be possible when larger numbers are available for analysis.

ACUTE POSTERIOR MULTIFOCAL PIGMENT EPITHELIOPATHY WITH CEREBRAI

\section{VASCULITIS}

G Stoll, C Althaus, R Unsöld. HeinrichHeine University, Düsseldorf, Germany

Acute posterior multifocal pigment epitheliopathy (APMPPE) originally described by Gass in 1968 (Arch Ophthalmol 80:177-85) is characterised by sudden, often binocular, blurring of vision. Usually the visual outcome is good and the disease has a self-limited natural course. Serious complications, however, may evolve from systemic manifestations which occur occasionally such as cerebral vasculitis. We report on two patients who developed neurological deficits four or six months after onset of APMPPE. MRI in the first patient revealed multifocal white matter lesions and angiography showed string-like arterial narrowing of all basal cerebral arteries typical of vasculitis. MRI performed at the onset of blurring of vision in the second patient was normal, while a single pontine lesion was found when he had developed hemiparesis. In both patients neurological symptoms promptly responded to treatment with corticosteroids and no relapses were observed under long term immunosuppression with azathioprine. These cases again stress that APMPPE is not a "benign" disorder. Further epidemiological studies are necessary to evaluate the frequency of neurological complications in APMPPE and to decide whether it is justified to start long term immunosuppression from the very beginning of the disease to prevent the development of harmful cerebral vasculitis.

\section{SARCOIDOSIS OF THE CENTRAL NERVOUS} SYSTEM

D Timmann, A Delcker, PM Faustmann. University of Essen, Essen, Germany

The diagnosis of CNS sarcoidosis is generally made on the basis of clinical features together with clinical and biopsy evidence of sarcoid granulomas in other tissues. A patient with strong evidence of neurosarcoidosis without granulomas elsewhere is described.

In 1988 a 35-year-old white woman developed abdominal pain, fever, and arthritis, and in 1989 peripheral lymphadenitis. In October 1989 she presented with cluster headache, and in 1990 with diffuse headaches, vomiting, blurred vision, and double vision. There is no history of genital or mouth ulcers. Neurological examination revealed bilateral papilloedema and bilateral abducens palsy. Brain MRI showed enhancement of the basal meninges and occipital lobes, CSF mild pleocytosis and positive oligoclonal bands, and biopsy of the meninges chronic inflammation. Erythrocyte sedimentation rate was slightly raised. Angiography, CT of the abdomen and chest, gallium scan, conjunctival and marrow biopsy, and laboratory findings (calcium, acetylcholinesterase lysocym, autoantibodies, HLA B5, HLA B27) were normal. Tuberculosis, crytococcosis, toxoplasmosis, syphilis, and brucellosis were excluded. Adminstration of corticosteroids and azathioprine led to clinical remission.

The diagnosis of neurosarcoidosis was made on the basis of chronic inflammation of the basal meninges, inflammatory CSF and the previous history in the absence of clear evidence of an infectious disease, lymphoma, Behçet's disease or vasculitis.

LONG TERM NEUROPHYSIOLOGICAL MONITORING IN HIV INFECTION: THE MIDDLESEX MRC COHORT STUDY

S Connolly, $\mathbf{H}$ Manji, $M$ Fell, $\mathrm{RH}$ McAllister, GB Griffin, CJ Fowler, SP Newman, IVD Weller, MJG Harrison. University College and Middlesex School of Medicine, London, UK

The MRC Cohort study aims to determine the nature of onset and natural history of the neurological complications of HIV infection. The Cohort members have regular six-monthly assessments consisting of a full neurological history and examination, detailed neurophysiological and neuropsychological testing, brain MRI and immunological investigations.

The neurophysiological tests include lower limb nerve conduction studies (sensory and motor), somatosensory evoked potentials (from posterior tibial nerves), magnetic stimulation of cortex and roots, and cognitive event-related potentials.

Ninety-two of the original 127 subjects have attended for their fourth visits. Twenty-three subjects were HIV-seronegative, 45 were asymptomatic HIV-seropositive and 24 were ARC/AIDS of whom 16 had progressed in the two years. The groups were matched in age, mood, alcohol, and drug use.

On cross-sectional analysis, the ARC/AIDS group differed significantly from the HIV-seronegative controls in a few test results (e.g. Sural SAP amplitude, P300 Latency-ANOVA $p<0.05$ ), but there were no significant differences between the asymptomatic HIV-seropositive group and the controls (ANOVA, p > 0.05). Furthermore, repeated measures analysis of variance has shown no evidence of significant deterioration in the asymptomatic HIV-seropositive subjects over the followup period. 
OCCURRENCE OF OLIGOCLONAL BANDS IN NON-INFECTIOUS NEUROLOGICAL DISEASES

U Wurster, P Lake, J Haas. Hannover Medical School, Hannover, Germany

While the development of oligoclonal bands (OB) in infectious neurological disease reflects the ongoing immune response in the CNS, their reported presence in other neurological disorders remains controversial and impairs their usefulness as a diagnostic test for multiple sclerosis. Our results are based on nine years experience with isoelectric focusing of unconcentrated CSF on polyacrylamide gels which have a higher resolution than agarose gels. Electroblotting and immunostaining of IgG confirmed the validity of the employed silver protein stain. However, faint alkaline bands could only be visualised with the (transparent) silver stain OB were found in disorders associated with immunological disturbances like the Sharp syndrome (3/12), lupus erythematosus (4/43), sarcoidosis $(1 / 19)$, but not in panarteritis nodosa $(0 / 14)$ or myasthenia gravis $(0 / 12)$. A local oligoclonal reaction was also noted in nine secondary brain tumors, two glicomas, two pinealomas and one chordoma. A few patients with facial pain, 3/23 with epileptic seizures, 4/50 with extrapyramidal movement disorders and $2 / 78$ with ALS also showed OB. Increasing the cut-of to four singular bands in the CSF, will slightly lower the sensitivity for multiple sclerosis by $2.5 \%$ to $96 \%$, on the other hand a considerable number of unexplained weak OB will be eliminated, e.g. 9/65 patients with cerebrovascular disease had more than one band, but only two surpassed the limit of four.

DO NEUROPHYSIOLOGICAL TESTS AND CT CONTRIBUTE TO THE DIAGNOSIS AND PROGNOSTIC EVALUATION OF LATE ONSET CEREBELIAR ATAXIAS?

G-P Huss, K Wessel, H Brückmann, D Kömpf. Medical University of Lübeck, Lübeck, Germany

Twenty-one patients diagnosed as suffering from autosomal dominant or idiopathic late onset cerebellar ataxia were investigated clinically, by means of neurophysiological tests (BAEP, blink reflex, SEP, MEP, VEP) and CT-scan. The study included an initial investigation and a follow-up examination on average 25.3 months thereafter (minimal eight, maximal 36). Patients could be divided into four groups: (a) patients with pure cerebellar ataxia (CA) after a course of four years minimum, (b) patients with pure cerebellar ataxia, who exhibited pathological neurophysiological tests at their last examination, (c) patients who upon initial examination clinically presented with pure $\mathbf{C A}$, but according to the last examination had developed a multisystem disorder, (d) patients with olivopontocerebellar atrophy (OPCA) presenting additional non-cerebellar signs of involvement. Conforming to a strict interpretation of pure cerebellar atrophy, Group 1 patients invariably exhibited normal neurophysiological tests at all times. Except for two individuals at the first examination all patients in Group 4 showed pathological changes in at least one of the neurophysiological tests.

The main point of this study is that individuals who according to clinical criteria were initially classified as CA but finally developed a multisystem disorder, already presented pathology in the neurophysiological tests on occasion of the initial examination (Group 3). The increasing frequency of pathology in the several neurophysiological tests together with the progression of the disease is obviously of prognostic significance. CT showed cerebellar atrophy without apparent involvement of brainstem structures in all patients with CA, most patients with OPCA had a visible additional atrophy of the brainstem. Three out of 10 patients with pure CA according to clinical criteria but with pathology in neurophysiological tests showed a brainstem atrophy on CT scans. The results demonstrate that neurophysiological tests and CT investigations performed at regular intervals are of diagnostic and prognostic value in late onset CA.

THE MEASUREMENT OF THE HUMAN

VESTIBULO-OCULAR REFLEX

IN THREE DIMENSIONS

E Koenig, M Fetter, D Tweed, H Misslisch. University of Tübingen, Tübingen, Germany

Until recently vestibular responses in humans had been evaluated by rotations around the vertical body axis in the uprigh body position, thereby stimulating only a minor part of the vestibular system, namely the horizontal semicircular canals Stimulation of the verticular semicircular canals and of the otoliths by rotation of the subject in the gravity field has only rarely been used and recording of eye movements under these circumstances has been of limited value when performed by the commonly applied electro-oculographic method (inability to measure torsional eye movements, unlinear responses with vertical eye movements).

To allow rotations around different axes with respect to the body a new apparatus was developed, which consists of a gimbal system providing a cardanic suspension of the human being with the head in the centre of rotation. The two outer rings of the cardanic suspension are motor driven, the two interior axes can be positioned in fixed angles. To exclude visible feed back about orientation in space the subject is surrounded by a light proof sphere the inner wall of which can serve as a projection screen for optokinetic experiments.

Eye movement recording is done in all three axes (horizontal, vertical and torsional) by the search-coil-method. The Helmholtz coils for the induction of the magnetic fields are attached to the oute surface of the visual protection sphere, so that they do not interfere with full field vision.

To evaluate the vestibulo-ocular reflex (VOR) in different directions five subjects were seated in the light proof sphere with eyes open in the dark and were rotated around the conventional longitudinal body axis, around an interaural axis and around a naso-occipital axis. Subjects were always positioned such that the rotation axis was parallel to the gravity vector, to avoid the changing otolith input. Stimuli were constant velocity rotations of $150 / \mathrm{s}$. The combination of three axes of stimulation and three axes of eye movement recording results in a $3 \times 3$ matrix, which gives a complete description of the human VOR Results computed so far, indicate that the human vestibulo-ocular reflex does not obey Listing's law and therefore differs from other types of human eye movement (saccades, pursuit).

CORRELATION OF NEUROPSYCHOLOGIC SCORES AND CEREBRAL BLOOD FLOW DECREASE IN PATIENTS WITH DEMENTIA OF ALZHEIMER TYPE

A Hartmann, K Broich, F Horn, M Gnad, L Solymosi, S Adam. University of Bonn, Bonn, Germany

In dementia of Alzheimer type (DAT) regional cerebral blood flow (rCBF) decrease is a consequence of neuronal failure. It has been claimed that decrease of rCBF primarily affects the parieto temporal area. It has not been settled whether rCBF reflects the clinical state in DAT. This prompted us to compare rCBF, neuropsychologic state and cerebral atrophy in patients with DAT.

In 53 patients with established dementia (DMS-III-R) DAT was diagnosed as being proven from the clinical point of view according to the criteria NINCDS. All DAT-patients with risk factors for vascular diseases except smoking were excluded. Neuropsychologic testing was performed using a standardised battery of 10 tests. rCBF was measured with the Xenon 133inhalation technique and 32 detectors arranged over both sides of the skull to measure the fast perfused tissue (primarily grey matter).

In patients with long lasting DAT (above three years, $n=24$ ) mostly the parietal lobe of both sides showed symmetric reduction of rCBF and in 30 of these an additional rCBF decrease in both frontal lobes. This correlated well with the severity of neuropsychological impairment. Patients with aphasia presented with $\mathrm{rCBF}$ reduction in the speech dominant hemisphere. There was no correlation of the intensity of tissue atrophy in cranial CT to rCBF and neuropsychological findings. In patients with shorter duration of the disease $(n=29)$ only 17 presented with a parietal $\mathrm{rCBF}$ reduction whereas the other 12 had a frontal rCBF decrease of asymmetric right-left distribution. These DAT-patients presented with the least neuropsychologic impairment.

It was concluded that in contrast to the literature DAT does not always start with an initial parieto-temporal lobe involvement. Patients with DAT may have rCBF reduction in the frontal lobe which (as observed in follow-up measurements) later on may spread to the parietal and temporal lobes. rCBF reflects the intensity of neuropsychological impairment better than atrophy as established by cranial CT.

EVENT-RELATED EEG POTENTIALS IN OLD AGE AND IN DEMENTIA OF THE ALZHEIMER TYPE

$\mathbf{R}$ Verleger, D Kömpf, W Neukäter. Medical University of Lübeck, Lübeck, Germany

Studies on event-related EEG potentials in normal ageing and in dementia have focused on the P3 component and often used auditory stimuli only. P3 latency was found to be delayed in normal ageing and to be additionally delayed in moderately 
demented patients. In the present study, components of the $\mathrm{N} 2$ complex (mismatch negativity and N2b) were analysed in the usual auditory oddball task in addition to P3, and a visual task was employed ("Push/Wait").

Comparing healthy elderly subjects to young adults, it was found in the oddball that the age delay was already present for mismatch negativity but further increased until P3's peak. In the Push/Wait task, the age delay had its onset after the occipital
P140 whereas the delay caused by reducing visual intensity was present before this component and did not interact with the age delay of P3. Within the elderly, P3 latencies correlated between the auditory and the visual task, and the common factor extracted from both latencies correlated with a test of short-term memory span. It is concluded that the age delay of P3 is not due to a delay of perceptual encoding but rather due to delayed memory processes in the elderly.

In a group of Alzheimer patients, with their mean IQ still in the normal range, P3 and mismatch negativity did not differ from the healthy elderly in the oddball task, but the patients' N2b was delayed. The main difference in the Push/Wait task was in an occipital P270, a component which was distinctly larger in the patients. It is suggested that both differences reflect the disintegration of patients' cognition: stimuli are perceived in a normal way but then a gap arises due to uncertainty what to do with the perceived event. 